Master's Thesis in Embedded Systems

\title{
An Energy-Harvesting Facade Optimization System for Built Environments
}

Atul Pandaravila Biju 



\title{
An Energy-Harvesting Facade Optimization System for Built Environments
}

\author{
Master's Thesis in Embedded Systems \\ Embedded Software Section \\ Faculty of Electrical Engineering, Mathematics and Computer Science \\ Delft University of Technology \\ Mekelweg 4, 2628 CD Delft, The Netherlands
}

Atul Pandaravila Biju

a.pandaravilabiju@student.tudelft.nl

25th October 2017 


\section{Author}

Atul Pandaravila Biju (a.pandaravilabiju@student.tudelft.nl)

Title

An Energy-Harvesting Facade Optimization System for Built Environments MSc presentation

30th October 2017

\section{Graduation Committee}

prof. dr. K.G. Langendoen (chair) Delft University of Technology dr. R.R. Venkatesha Prasad Delft University of Technology

prof. dr. Elmar Eisemann

Delft University of Technology

Daily Supervisor

dr. Chayan Sarkar

TCS Research, India 


\begin{abstract}
Daylighting is the immediate exploitation of solar energy in the form of natural lighting and plays an integral role in minimizing the energy footprint of a building. Smart daylighting enables us to design buildings that provide comfort and energy savings.

This work proposes a dynamic facade system for buildings which aims to maximize user comfort while simultaneously maximizing energy savings by harvesting solar energy optimally. The solar panels on the facade can harvest the highest amount of energy when it is positioned perpendicular to the suns rays. However, this may result in unsatisfactory lighting conditions inside the room and the problem is approached as a bi-objective optimization problem. This work is a preliminary exploration of the concept of smart skins for buildings that autonomously regulates light while harvesting solar energy, contributing to the creation of the future of sustainable buildings. The primary focus of this research work revolves around building a conceptual model, formulating an optimization problem, developing a control algorithm, iFOS, and then evaluating it. Data was simulated using advanced simulations to evaluate the dynamics of light indoors.
\end{abstract}

Two benchmarks were created to evaluate the algorithm against, one where the system works towards maximizing user comfort indoors, and the other, where the system works to maximize the energy harvested by the facade. Upto $8 \%$ increase in the energy harvested was achieved with minimal loss in user comfort in the use case evaluated. The average energy figure for The Netherlands in the summer months is about $5 \mathrm{kWh} / \mathrm{m}^{2} /$ day, which makes the total energy that can be captured at $20 \%$ efficiency to be about 750 $\mathrm{kWh}$ per day. The algorithm is found to work the best when the desired light level to be maintained indoors lies in the range $[400,600]$ lux. 


\section{Lokah Samastah Sukhino Bhavantu लोकाः समस्ताः सुखिनो भवन्तु}

"May all beings everywhere be happy and free, and may the thoughts, words and actions of my own life contribute in some way to that happiness and to that freedom for all."

-Indian Mantra 
"Dedicated to my parents."

"To my mother, for showing me who I really am, and to my father for showing me who I really can be." 



\section{Preface}

The past 10 months have been a huge learning ground for me, both academically, as well as personally. Reflecting back, there were definitely tough times, but on the bright side, there were so many new frontiers that I explored, never realizing they existed. I am quite convinced that climate change is truly a global phenomenon that has to be actively discussed, engaging the whole world. Through this thesis, I got to read many interesting articles that addresses it by presenting scientific data from scientists across the world. The things I've seen and read have definitely had a big impact in me empowering myself to contribute to the cause.

That said, it is now time to convey my heartfelt gratitude to the people who have made this journey possible. None of this would have been possible without the constant support from two individuals I've called "Acha" and "Amma" for the past 25 years. They spent most of their prime years nurturing and moulding me into who I am today. Talking about family, none of this would have been possible without María, who constantly supported me emotionally as well as being my MATLAB instructor, planning time-crunch recipes and more. I don't know where to start about VP, my supervisor. He has been one of the most dynamic people I've ever come across, and if there's one thing he's taught me well, it is to provocatively poke my own thoughts before reaching conclusions. I truly am grateful to him for all the support. Special mention goes out to Chayan, who supervised me, from half the way across the world. Koen, for his honest reviews and feedback. To Nikos, for being that person who I can always turn to for any random thought that needs validation, for a second opinion and more.

To every other person, who has had a part to play in this, thank you for everything. The 9th Floor in EWI will always have a special place in my heart, for being "gezellig". Thank you for teaching me important lessons that will help me in the forthcoming journeys in life.

Delft, The Netherlands

25th October 2017 



\section{Contents}

Preface vii

1 Introduction $\quad 1$

1.1 Problem Statement . . . . . . . . . . . . . . . 3

1.2 Approach ...................... 5

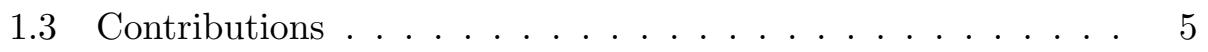

1.4 Organization ..................... 6

2 Background $\quad 7$

2.1 Facades . . . . . . . . . . . . . . . . 7

2.2 Daylight Harvesting . . . . . . . . . . . . . . . . 10

2.3 Sun Position . . . . . . . . . . . . . . . . . . 12

2.4 Solar Irradiance . . . . . . . . . . . . . . . . . . . 13

2.4 .1 Direct Normal Irradiance . . . . . . . . . . . . . . . 13

2.4.2 Diffuse Horizontal Irradiance . . . . . . . . . . . . . 14

2.4.3 Global Horizontal Irradiance . . . . . . . . . . . . 14

2.5 Plane of Array Irradiance . . . . . . . . . . . . . . 15

3 System Overview $\quad 17$

3.1 Structural Design . . . . . . . . . . . . . . . 17

3.2 Conceptual Model Overview . . . . . . . . . . . . . . 20

3.3 High-level Workflow . . . . . . . . . . . . . . . . 21

4 Discovering Light Dynamics and Energy Characteristics 23

4.1 Facade Test-Bed . . . . . . . . . . . . . . . . . . 24

4.2 Simulating the Environment . . . . . . . . . . . . 25

4.3 Daylighting Analysis . . . . . . . . . . . . . . . . . . . . 29

4.4 Solar Energy Calculations . . . . . . . . . . . . . 32

5 Optimization Formulation and Algorithm 35

5.1 Problem Definition . . . . . . . . . . . . . 35

5.2 Problem Formulation . . . . . . . . . . . . . 36

5.3 FOS Controller . . . . . . . . . . . . . . . . . . 39 
6 Evaluation $\quad 41$

6.1 Facade Test-Bed . . . . . . . . . . . . . . . 41

6.2 Simulations of the conceptual facade . . . . . . . . . 45

6.3 Proposed Algorithm: iFOS . . . . . . . . . . . . 50

6.3.1 Benchmark 1: Maximum Comfort . . . . . . . . . 50

6.3.2 Benchmark 2: Maximum Energy Harvested . . . . . . 52

6.3 .3 iFOS Optimized . . . . . . . . . . . . . 53

6.3 .4 Impact of iFOS . . . . . . . . . . . . . . . 56

7 Conclusion and Future Work $\quad 59$

$\begin{array}{ll}\text { A Comparison plots for } c=10 & 61\end{array}$

B Comparison plots for $\mathrm{c}=2.5 \quad 63$ 


\section{Chapter 1}

\section{Introduction}

H

I UMAN beings began building with simple forms of construction to shelter themselves from the sun, wind and rain. Since the ancient times, settlements in temperate climates have preferred massive wall structures that protected the inhabitants from the harsh sun during the day and cold winds during the night. However, around the turn of the $20^{\text {th }}$ century, these massive walls gave way to lighter structures made of cast iron and steel, thus introducing the world to curtain-wall facades. During this developmental phase of modern buildings, engineers and architects were more interested in the aesthetics and the formal aspects of this new architecture rather than in its energy performance. As a result of this, most of the buildings built during this era had unacceptable indoor comfort conditions, poor natural ventilation, and exorbitant energy consumption. High-rise buildings were the epitome of these developmental strides in architecture. A serious concern among these developments was the inadequate ability to control the indoor environment because of their high exposure to the sun [1].

The concept of energy efficient lighting aims at decreasing the energy signature of the built environment while striving to maintain or improve the existing illumination quality. This concept of quality however includes multiple aspects and there are no set of concepts to make an overall judgement of what is called lighting quality. For us humans, our visual sense has developed over millions of years under the influence of daylight and even now with the advent of artificial lighting, we perceive and experience daylight as 'natural'. Daylight differs immensely from the light given out by artificial light sources as it has a continuous spectrum, contrary to most artificial lighting sources available today. It also has a constantly varying intensity and wavelength composition, which has been proved to have biological, visual, and emotional aspects. These aspects include health, diurnal rhythm, visual comfort, task 
performance, and perception of colours and space [2]. Therefore, daylighting is considered an established part in the integral design process of a modern building. It strives to optimize the availability of glare-free, natural daylight into the interior of the building. The term 'daylighting' is predominantly used in the context of commercial buildings in which the hours of daylight availability and building occupation largely overlap [3].

Energy from the sun is by far the largest source of energy on Earth, around $3.9 \times 10^{24} J$ of solar energy reaching our surface annually. In perspective, this is about 10,000 times the global energy demand [4]. Environmental concerns are ever more important now than ever in the history of mankind. Presently, the world is consuming fossil fuels at an alarmingly high rate. The figures of emissions from the industrial world for 2015 was a whopping 36.2 billion tonnes of carbon dioxide along with the fact that 2015 was the hottest year since 1880 [5].

Solar energy is an important source of energy with the potential to satisfy many challenges today's world faces. In 2015, solar energy was the fastest growing energy sector with a $33 \%$ rise, with the environmental advantages being the main driver in this growth [6]. Solar energy is a safe alternate which can replace current fossil fuels like coal and gas for electricity generation that produce air, land and water pollution. There has been convincing evidence from the scientific community that the phenomenon of climate change is very much real. Solar energy can restrict climate as it produces no carbon emissions. The greatest attraction of this renewable source of energy is that it can be produced on a small-scale directly by the end consumers in contrast to large, centralized conventional energy sources controlled by huge corporations. However, the main concern over solar power is the initial investment of purchasing and installing the panels. This cost will only come down once the technology becomes widespread and the volumes are high. Yet, the cost savings over time and the positive impact on the environment make it an increasingly attractive alternative for residential and commercial buildings alike. 


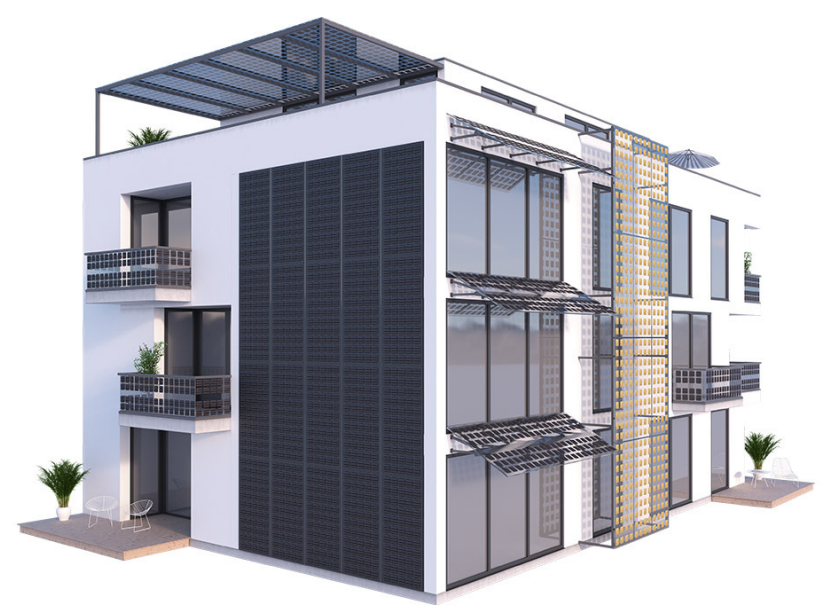

Figure 1.1: A typical example of BIPVs.

BIPVs ${ }^{1}$ are photovoltaic materials that are used in place of conventional building components like windows or facades and are usually integrated into the buildings to harvest energy as well [7]. An application of the concept is shown in Figure 1.1. Adding dynamic parts that move along the day, following the sun can increase the energy harvesting potential as it optimizes the angle of incidence of the incoming rays of the Sun with the solar panels.

\subsection{Problem Statement}

High-rise buildings experience direct sunlight causing sub-optimal lighting conditions to their indoor environments due to glares or excessive light. Traditionally, blinds or facades are used to solve this problem but they require manual adjustments throughout the course of the day. The dependency on artificial lighting is also increased as the natural light is not put to optimal use. Lighting accounts for nearly $43 \%$ of the energy expenditure in office buildings [8] and this figure can be decreased with energy-efficient designs of buildings, where the next generation facades play a pivotal role by maximizing the usage of daylight.

High-rise buildings also have a small roof area compared to their floor area. Often, the roof houses ventilation ducts and cooling towers, further decreasing the usable area for harvesting solar energy. A solution to this challenge is to integrate solar energy harvesting components into the sides of the building. Without dynamic elements adjusting tilt angles of these solar cells to the sun's incident rays, the efficiency would be low. Although this depends

\footnotetext{
${ }^{1}$ Building integrated photovoltaics
} 
on seasons and location, a dynamic system can increase efficiencies significantly. Further, rapid changes to the position of the facade to control daylight has been shown to cause irritation to the users [9] and thus it has to be designed in a way that causes minimal discomfort to the user.

Yun et al. proposed an index, effectiveness of a PV Facade (PVEF), that has been developed to evaluate the overall energy performance of a PV facade with regard to the proportion of useful daylight that may displace the use of electric lighting, and the electricity generated by the PV modules to the heating and cooling energy consumption within a building [10]. Although factors such as ventilation and thermal effects were evaluated, the possibility of the performance changing with varying tilt angle of the solar panel along the course of the day was not studied.

Following the invention of large area glass, architects have increasingly employed the material in facades to achieve transparency, for daylight access and views to the exterior. Ever since, numerous technologies have been implemented to achieve better lighting in high-rise buildings. However, stateof-the-art technologies remain limited in their comprehensive treatment of various concerns like visual and thermal comfort, energy signature, extent of view preservation and architectural design implications. Electrochromic technology has gathered a huge success over recent years, but it suffers from material dependent limitations, like slow switching times, sometimes as high as 80 minutes under low temperatures [11]. Due to this, their application has mostly been limited to warm climates, where they have been predominantly tested. Even under optimal conditions, electrochromics still require about 5 minutes to change states. This exceeds the occupants' expectations for response times, thereby preventing most levels of interactivity with the system.

Following the above mentioned motivation and the limitations in the existing work, this thesis targets at solving the following research problem:

To design a modular facade and develop a smart control algorithm that maximizes both the user's comfort level and the facade's energy harvesting capabilities, ensuring user interactivity. 


\subsection{Approach}

The facade optimizes light intensity for users by controlling the daylight entering the room while lowering the dependency on artificial lighting and optimizes the energy harvested by tilting itself along with the embedded solar cells as close as possible to the perpendicular rays of the sun. The algorithm controlling the facade has to predict the solar energy generating capabilities of the facade. The proposed system ensures even distribution of daylight in office spaces and uses artificial illumination by incorporating light fixtures into it so that the desired light level is adequate at all times of the day, adjusting for weather and seasons. This is achieved while also maximizing the energy that is harvested from the sides of high-rise buildings, which is almost absent in today's world. The proposed algorithm gets the user-in-the-loop by incorporating parameters that the user inputs, giving the user a sense of control. If multiple users are present in the room, the algorithm works in such a way that all the users are content with the way the system works.

However, critical issues like rapid changes in the daylight levels caused due to cloud movements and the extent to which shading affects the efficiency of solar cells remain unaddressed in this research work.

\subsection{Contributions}

This thesis presents the concept of $\mathrm{FOS}^{2}$, which is an energy harvesting facade optimization system for built environments and, iFOS, which is the algorithm that optimizes the movement of the facade, enabling optimized user comfort and maximizing the energy harvested while doing so. The author's contribution can be divided into the following:

- An algorithm called iFOS is proposed, which controls the movement of the facade, optimizing user comfort and maximizing energy harvested simultaneously.

- Proposed algorithm, iFOS, was analyzed and evaluated against two benchmarks: one where the system focuses solely on maximizing user comfort and the other where it solely focuses on maximizing energy harvested.

\footnotetext{
${ }^{2}$ Facade Optimization System. In Greek, fos(phos) means "Light".
} 
- A simulated testbed was created to analyze the dynamics of light in the room and study light behaviour at any time of the year, for various sky conditions.

- A small-scale testbed was created from scratch to validate the reliability of the simulation.

- Measurements were performed to observe illuminance in the room for various tilt angles of the facade and differing sky conditions for selected days of the year.

\subsection{Organization}

This research work is divided into seven chapters. The next chapter, Chapter 2, lays some basic groundwork, helping the reader understand some key concepts used in this work, with the works related to each topic also analyzed. The system design and key points about the concept are explained in Chapter 3. Chapter 4 discusses the simulation setups and the various plugins used. A mathematical formulation of the conceptual facade is modelled in Chapter 5, followed which the optimization algorithm, iFOS is proposed. Chapter 6 discusses the data from the simulations and evaluates the proposed algorithm iFOS against two benchmarks. Chapter 7 discusses some important conclusions and lays the ground for future work. 


\section{Chapter 2}

\section{Background}

This chapter presents some background knowledge on facades and their design elements. It also discusses some existing daylight harvesting models and their working principles. Further, insights into efficient solar energy harvesting are presented along with some key concepts and terms.

\section{$2.1 \quad$ Facades}

The problem of engineering present day facades is one that is taken up with utmost importance in the construction industry. Over the last few years, an increasing number of buildings have been incorporating facades into their designs. Although they look aesthetically more pleasing than the concrete jungles that came with the industrial revolution, facades serve a far more important purpose than just covering the beams beneath. A smartly designed facade can help reduce the energy footprint, improve daylighting and pave way for better airflow in buildings, thus serving more than just pleasing the eye. Research suggests that facades can add up 10\% to $35 \%$ of the total cost of buildings [13]. However, this cost is deemed worthwhile as over time the costs balance out with savings from energy costs, thus addressing the cause of sustainability.

By definition, a modern facade, also called a building envelope, is an independent frame assembly with self-sufficient components that do not brace the building structure [14]. The building envelope is the first line of defense against environmental and physical exposure based on its intrinsic design and location. Figure 2.1 illustrates the design requirements of modern-day facades. 


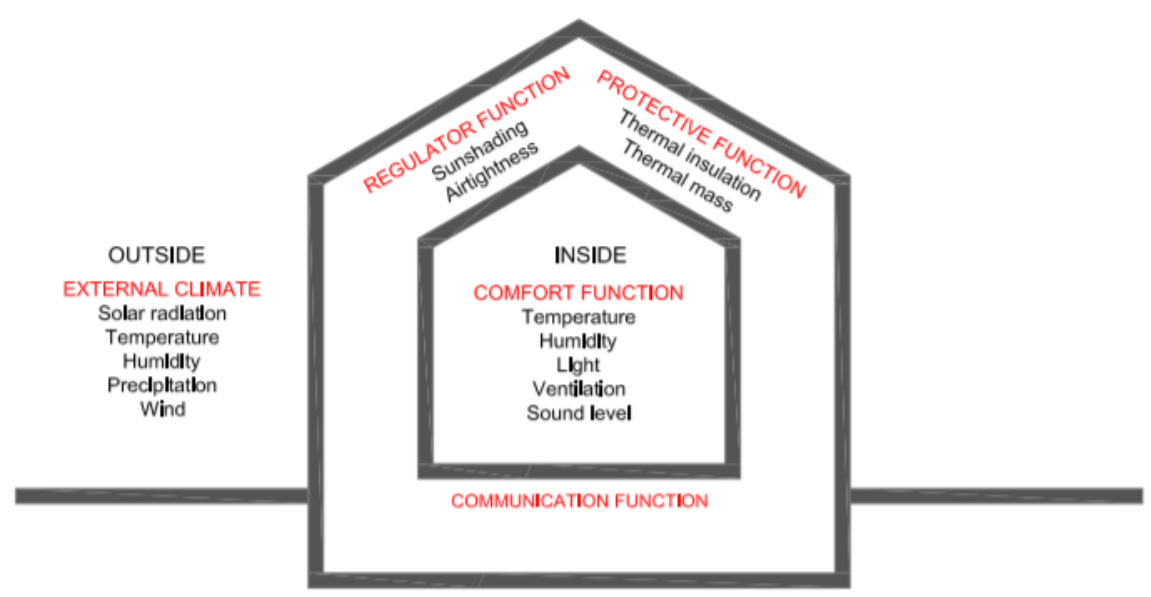

Figure 2.1: Conditions and requirements of a facade as illustrated by Arch. G. Paoletti [12].

Given its unique location, there is high expectation of performance for this building element. It's primary goal is to act as the first line of defense against environmental threats to the building as well as play a vital role in the transmittance of light into the interior of the building. Figure 2.2 shows an architect's vision for the future of facades and their holistic design methodologies in treating facades as an integral, connected part of future buildings. The scope of this thesis work is to explore the possibility to autonomously control and maximize the daylight entering buildings.

The latent potential of climate adaptive building shell (CABS) was explored and quantified by the authors in [15]. They state that the role of optimization in CABS is underexplored and therefore the true value and potential of such a system can only be guessed. Also, in the use case presented, it was shown through simulations that an energy reduction of about a factor of two wasachieved, compared to the best performing static building shell design while maintaining equivalent comfort conditions. The study was concerned with thermal comfort indoors but the authors conclude by saying that in real-life scenarios, the performance of such a shell will be determined by more aspects and they considered the extension towards daylighting viable.

Cole et al. created a framework which contrasts the merits and consequences of automated and human control strategies [16]. It is stated that an unintended economic consequence of relying on human intelligence is a higher level of worker retention. This is due to an increased inhabitant connection with the exterior environment, a heightened sense of responsibility and greater 


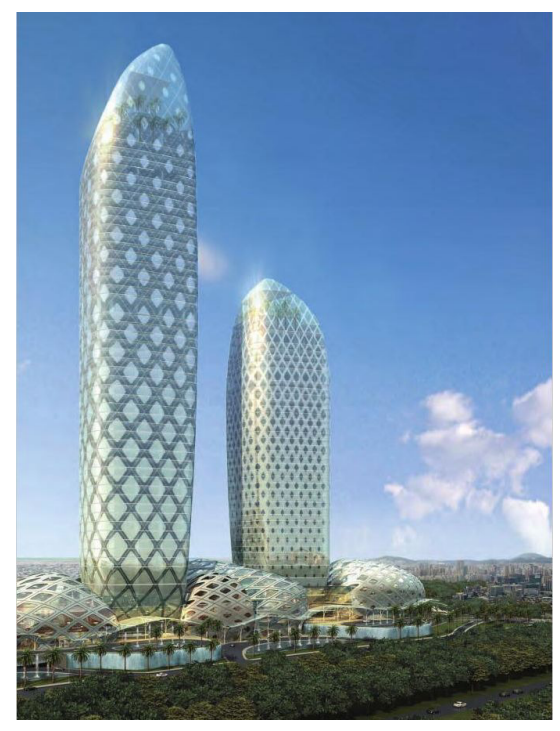

Figure 2.2: An architect's rendition of facades in the future.

comfort levels indoors. They conclude by stating that it is important to introduce technology that promotes interactions among humans rather than interactions with computing technologies, ignoring the user in the process.

S. Kang et al. analyzed the electric energy harvesting efficiency of venetian blinds according to their design parameters using photovoltaic modules in [17]. Through their study, they were able to prove that the variables for estimating the energy harvested by the blinds were solar azimuth, inclined angle of the blind, area of photovoltaic material and distance of the blind from the outer window. They also proved that the inclination angle and the distance from the window had the most significant influence in the shading phenomena. Although their analysis is primarily concerned with the effect of ventilation indoors on the efficiency of energy harvested, this work gives important insights into energy harvesting structures for buildings.

From the research documented, it is clear that there is a substantial potential in harvesting energy from facades. However, there are very few research works looking to optimize the problem of comfort versus energy efficiency, which this research works aims at addressing. 


\subsection{Daylight Harvesting}

Daylight harvesting is the term used to describe methods of maximizing the amount of natural sunlight entering a building. The value of daylight harvesting is simple: as daylight levels increase, artificial light levels can be decreased to maintain the desired amount of light indoors, thus saving a vast amount of energy. Daylight harvesting systems strive to automate this process and remove the element of manual control. Sensors constantly measure the indoor and outdoor lighting conditions and adjust lights according to the situation and the predefined relationship between the two positions. The control of daylight harvesting systems can be classified into two categories of systems:

\section{Open-loop}

Open-loop systems only measure the daylight and do not consider the artificial light contributions. The sensors are not exposed to any artificial lighting and usually reads out illuminance values outdoors or on a window facing outwards. It is called so since there is no feedback from artificial lighting indoors and hence is an open loop.

\section{Closed-loop}

Closed-loop systems measure the combined contribution of daylight and electric lighting in indoor spaces. The sensors pick up the contributions of artificial and daylight and hence a change in any of them is fed back into the system, creating a closed loop. With this, real-time measurements of light levels becomes possible and this technique is considered to be more accurate than an open loop. Typically, a space is divided into zones, each with a sensor, and are controlled together in a particular configuration. This is very helpful for applications where a specific target light level is to be maintained.

For daylighting systems, a target illuminance level has to be set along with a set of criteria defining how the system responds to maintain the desired configuration. To enable this, typically the additional parameters that have to be set are:

- Deadband- The extent of change from the target level of illuminance that the system tolerates. This essentially creates a range around the target level that is acceptable. It is usually set in terms of a particular lux value or as a percentage of the target level. 
- Delay Time- The amount of time the system waits to adjust the light levels when the light falls out of the deadband. This is to prevent the system from adjusting light levels due to short-term events like a passing cloud or any other short-term environmental cause.

- Fade Time- The amount of time the system takes to adjust the light levels once a change is initiated. This is included in the design to cause minimum disturbance to the users.

J. Lu et al. presented a concept of smart daylight harvesting that senses incoming daylight in a building in order to achieve stable task lighting intensity despite the unstable natural light source [18]. Their system used a combination of automatic modelling and daylight prediction techniques to predictively control the window transparency. An ingenious method of detecting sky cloudiness was also proposed. However, there was no connection of the daylight levels with other parameters of comfort like thermal or visual comfort and the system solely worked to keep the lighting condition close to a defined level.

R. Kumar proposed an algorithm for daylight harvesting in a private office in [19]. The problem of daylight harvesting was studied in an office space with adjustable electric lights and blinds. Data fusion algorithms were proposed that employ machine learning to compute electric light and daylight component of the total illuminance indoor. The experiments have a drawback as they were performed in a highly constrained environment and this may cause the algorithm to malfunction in a normal environment. Thus, it is learned that during the learning phase, all experiments are to be conducted in a normal environment.

Y. Wang et al. presented an adaptive lighting control system that can automate a building without the need for any custom designing [20]. They prove that the lighting control problem built using non-linear integer programming is NP-hard and propose a heuristic algorithm to solve the problem to compute approximate optimal solutions. The system was found to be still in its nascent stages and relies heavily on databases of data learnt over time. Hence, for the system to be robust, it would need to learn more and store them in datasets.

Y .Yoon et al. developed an annual daylight simulation algorithm for prediction of indoor daylight illuminance.[21]. 


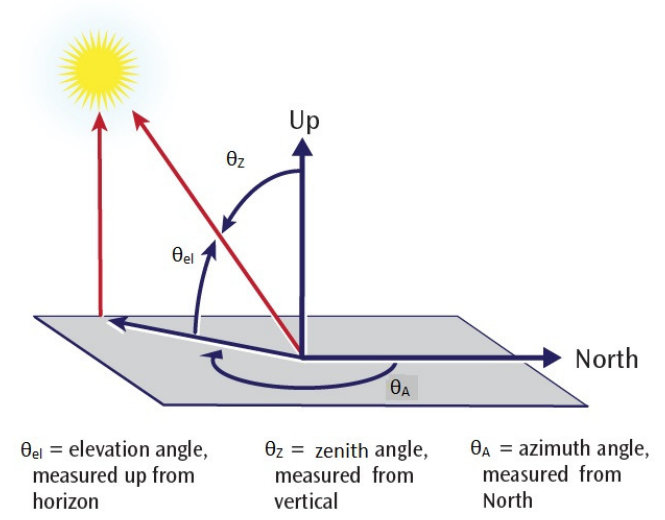

Figure 2.3: Conventions of the solar angles [22].

\subsection{Sun Position}

The modelling of the performance characteristics of the photovoltaic components involves accurate knowledge of the relative position of the Sun with respect to the system. The position of the Sun in the sky is a function of both time and the geographical location of the user on the surface of the Earth. With this knowledge, it is possible to define a few conventions that will be used. The conventions commonly used to describe the solar positions are:

- Zenith angle $\left(\theta_{Z}\right)$

- Azimuth angle $\left(\theta_{A}\right)$

- Elevation angle $\left(\theta_{e l}\right)$

Figure 2.3 describes these conventions and it is to be noted that $\theta_{e l}$ is $90^{\circ}$ $\theta_{Z}$. In order to calculate their values, the declination angle $\theta_{d}$ is to be computed [22]. The declination angle $\theta_{d}$ is the angle between the Earth's axis of rotation and the plane normal to a line connecting the centers of the Earth and Sun. The value is defined as $0^{\circ}$ by convention at solar noon when the sun is at its highest point in the sky. A simple approximation of this in degrees is given by:

$$
\theta_{d}=\frac{23.45 \pi}{180} \times \sin \left(2 \pi \frac{(284+n)}{365}\right)
$$

where $n$ is the day of year. 
Now, the values for zenith and azimuth angles are computed using the Solar Position Algorithm [23]. The user needs to specify the date of the computation and this is converted to the corresponding Julian Day. The method is purely mathematical and due to the complexity of the calculations, the algorithm is explained step-by-step.

1: Input: Date, Time, Latitude, Longitude.

2: Calculate the Julian and Julian Ephemeris Day, Century and Millenium.

3: Calculate the Earth heliocentric latitude (B), longitude (L) and radius vector $(\mathrm{R})$.

4: Calculate the geocentric latitude and longitude.

5: Calculate the nutation in longitude and obliquity. Then compute the true obliquity of the ecliptic.

6: Calculate the geocentric sun right ascension and sun declination.

7: Calculate the observer local hour angle $\mathrm{H}$.

8: Output: Zenith angle, Azimuth angle.

Algorithm 1: Solar Position Algorithm (SPA)

\subsection{Solar Irradiance}

To model photovoltaic performance models, weather and irradiance data are used as input. These data can be directly measured or simulated using stochastic models. Irradiance is an instantaneous measurement of solar power over some area and its unit is watts per square meter $\left(W / m^{2}\right)$. For practical purposes, irradiance is expressed in different components. For each measurement, there has to be a collection plane that is defined. The collection plane may be oriented in any direction to the sun. If extraterrestrial, all irradiances are direct due to lack of an atmosphere to cause scattering. The following are the main components of the irradiance measurements:

\subsubsection{Direct Normal Irradiance}

Direct Normal Irradiance (DNI) is defined as the amount of solar radiation received per unit area by a surface that is always held normal to the incoming rays from the sun's position in the sky. It is usually measured directly with an absolute cavity radiometer and is considered the most accurate method to measure it. But since these instruments are not designed for continuous 


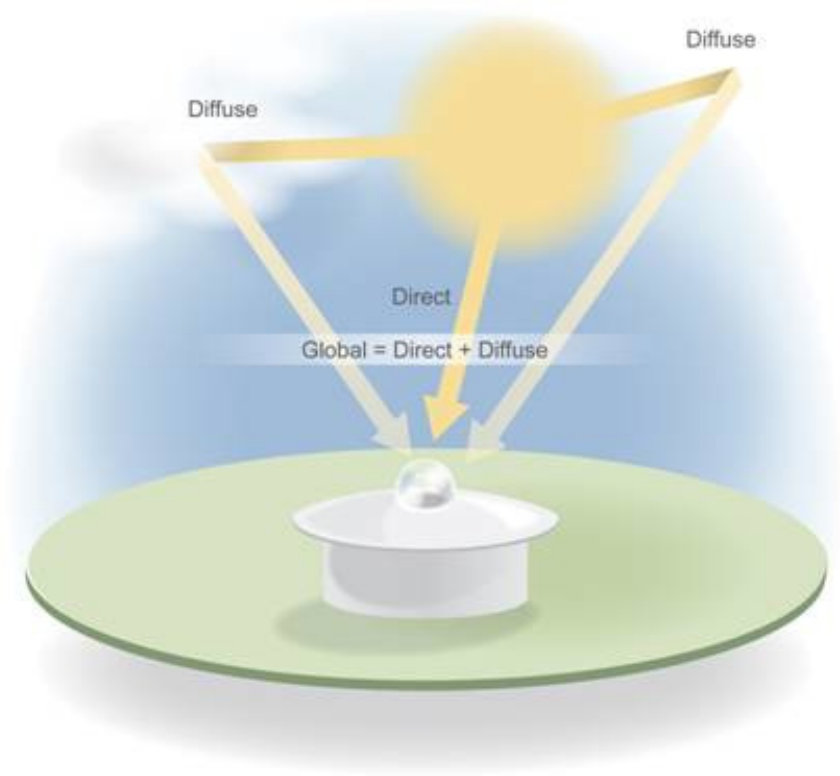

Figure 2.4: Direct.

outdoor use, the primary method of measurement of DNI is with an instrument called pyrheliometer. They use thermopile sensors at the base of a light-collimating tube which allows a field of view. If direct measurements are not available, DNI can be calculated via co-planar measurements of the diffuse and total radiation. There also exists models that can estimate DNI values from environmental factors, some of which are DISC and DIRINT models respectively.

\subsubsection{Diffuse Horizontal Irradiance}

Diffuse Horizontal Irradiance (DHI) is defined as the amount of solar radiation received per unit area by a surface that does not arrive on a direct path from the sun, but scattered by particles in the atmosphere of the Earth. It is measured with means of a pyranometer. DHI values can either be measured directly or indirectly using global and direct irradiance.

\subsubsection{Global Horizontal Irradiance}

Global Horizontal Irradiance (GHI) is defined as the total amount of solar radiation received per unit area by a surface. This metric is of particular interest to photovoltaic installations and it includes both DNI and DHI. It 
is represented mathematically as:

$$
G H I=D H I+D N I \cdot \cos \left(\theta_{Z}\right)
$$

Data used for computations are from the publicly uploaded datasets of Koninklijk Nederlands Meteorologisch Instituut (KNMI). The data set consists of GHI, DNI and DHI values recorded every hour.

\subsection{Plane of Array Irradiance}

A fundamental step in calculating the irradiance is to transpose the irradiance onto the plane of the array (POA) of solar cells. This step is critical for modelling the performance of a photovoltaic system. The steps in this direction include defining the orientation of the array, which can be fixed or dynamic and estimating the contributions of the direct beams and diffuse irradiance components. The POA irradiance is dependent on several factors including sun position, array orientation, irradiance components, Ground Surface Reflectivity (albedo) and shading.

Mathematically, POA irradiance, denoted as $E_{P O A}$ is defined as,

$$
E_{\mathrm{POA}}=E_{b}+E_{g}+E_{d}
$$

where $E_{b}$ is the direct beam component, $E_{g}$ is the ground-reflected component and $E_{d}$ is the sky-diffuse component.

Before discussing the above components in detail, it is important to look at the definition of another critical term, angle of incidence (AOI). It is defined as the angle between the sun's rays and the photovoltaic array at any particular instant of time and can be determined by the formula:

$$
A O I=\cos ^{-1}\left[\cos \left(\theta_{Z}\right) \cos \left(\theta_{T}\right)+\sin \left(\theta_{Z}\right) \sin \left(\theta_{T}\right) \cos \left(\theta_{A}-\theta_{A, \text { array }}\right)\right]
$$

where $\theta_{Z}$ and $\theta_{A}$ are the solar zenith and azimuth angles respectively, $\theta_{T}$ and $\theta_{A, \text { array }}$ are the tilt and azimuth angles of the array respectively. Azimuth angle follows the convention of the value in angles east of north, e.g. North $=0^{\circ}$, East $=90^{\circ}$, West $=270^{\circ}$. Array azimuth angle follows a similar convention and an array of solar cells pointing South has an array azimuth of $180^{\circ}$.

The direct beam component of irradiance $E_{b}$ is calculated by adjusting the DNI with the AOI, i.e

$$
E_{b}=D N I \times \cos (A O I)
$$


The ground-reflected component $E_{g}$ is the radiation that reaches a surface on being reflected from the ground. It is calculated as a function of the reflectivity of the ground surface, known as albedo and the angle of tilt of the surface, which in this case is $\theta_{\text {fos }}$, the tilt angle of the facade. This is determined by the formula:

$$
E_{g}=G H I \times \text { albedo } \times\left(\frac{1-\cos \left(\theta_{\mathrm{fos}}\right)}{2}\right)
$$

The sky-diffuse component $E_{d}$ is typically divided into many sub-components. These include:

- isotropic component, representing the uniform irradiance.

- circumsolar diffuse component, representing the forward scattering of radiation in the area around the sun.

- horizon brightening component.

A majority of the published models use various semi-empirical approaches for estimating the combination of these components. Since this research work will not have to estimate this value and rely on data from KNMI, only the isotropic sky-diffuse model needs to be understood. This is the simplest of the POA sky-diffuse models and forms a strong foundation upon which other models are built. The model assumes that the diffuse radiation from the sky dome is uniform across the sky. The sky-diffuse irradiance $E_{d}$ is calculated as a fraction of the measured DHI as:

$$
E_{d}=D H I \times\left(\frac{1+\cos \left(\theta_{\mathrm{fos}}\right)}{2}\right)
$$

where $\theta_{\text {fos }}$ is the tilt angle of the facade. 


\section{Chapter 3}

\section{System Overview}

The previous chapter discussed the most important concepts that defines this research work. Through this chapter, we present and discuss the system model of FOS. Furthermore, the architectural details are presented in order to give the reader a holistic view of the work. Advantages and limitations of design choices are also discussed. First, we look at the structural design of the system.

\subsection{Structural Design}

The conceptual model of FOS consists of multiple flaps connected to a frame that is fixed outside the windows of high-rise buildings. The flaps are capable of folding themselves, leaving spaces for light to enter the building. This is controlled by a servo motor that is connected to the flaps through a rack and pinion mechanism. The flaps open and close in an umbrella-like motion and a stepper motor controls the angle through a connected gear system.

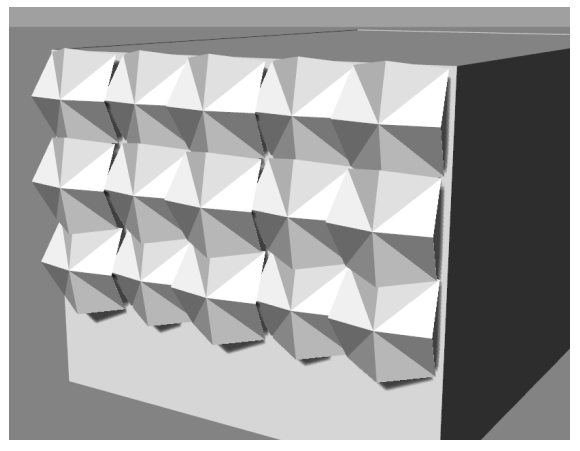

(a) Fully closed

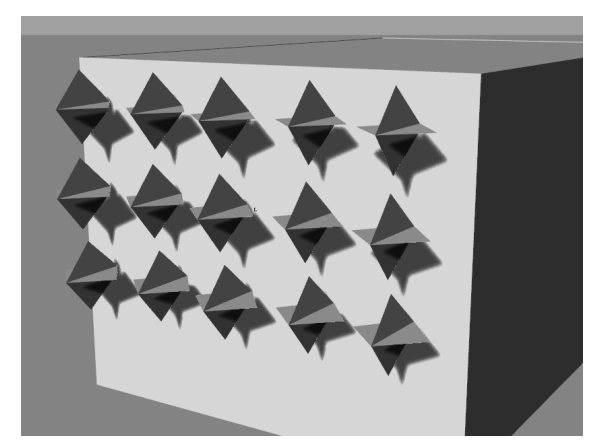

(b) Fully open

Figure 3.1: Renders of the conceptual FOS. 
For better interpretation and understanding of the system, a block of the system is considered. A block consists of 15 flaps moved together by a servo motor. Figure 3.1 shows the two extreme orientations of a block of FOS, one fully closed and the other fully open when placed outside the windows. The variable that is controlled to enable a change in the orientation of the flaps is the tilt angle of the system $\theta_{\text {fos }}$.

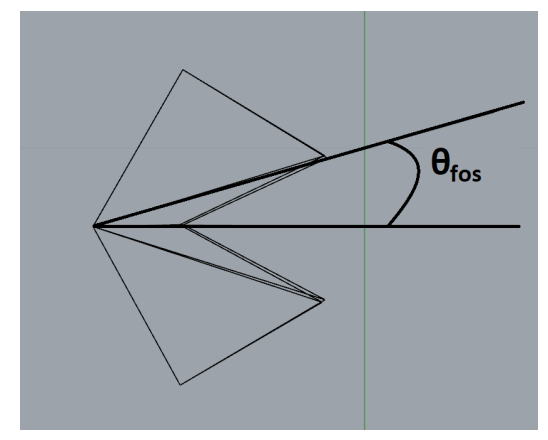

Figure 3.2: Lateral view of a flap describing the tilt angle $\theta_{\text {fos }}$.

Constrained by the design, the tilt angle of $\theta_{\text {fos }}$ varies from $75^{\circ}$ when fully closed to $5^{\circ}$ when fully open. Figure 3.2 shows the representation of the tilt angle $\theta_{\text {fos }}$. This range was chosen after research suggested that the optimum tilt angle for solar panels for Delft, The Netherlands across the year fell within this range. The initial version, as shown in Figure 3.3, had a range of tilt angles from $0^{\circ}$ to $45^{\circ}$. MATLAB simulations across the year suggested that increasing the range of tilt angles will also increase the efficiency of harvesting energy.
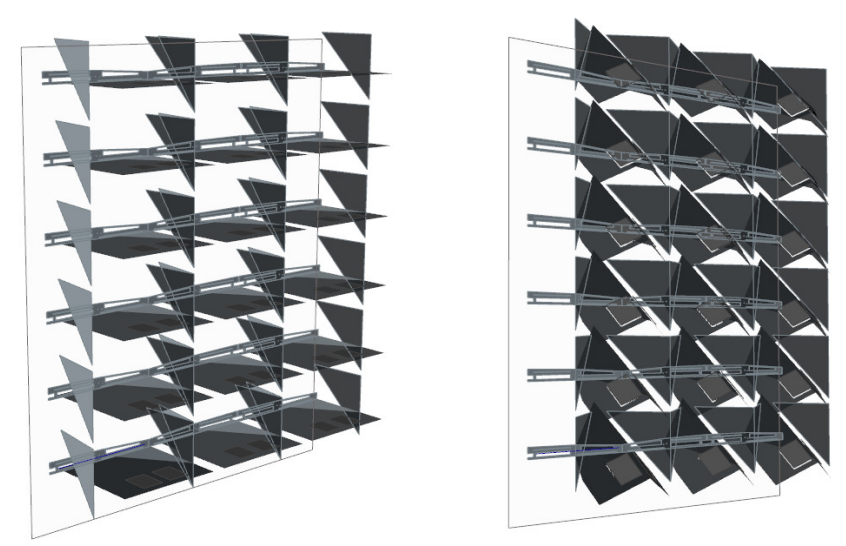

Figure 3.3: Initial design of FOS when fully open and closed respectively. 
The nature of the design also meant that in a single block, half the flaps had a particular azimuth angle in addition to the tilt angle and the other half had another azimuth angle. However, this was not seen as a flaw in the design but rather the design was iterated to make all the solar panels have identical tilt angles for simplicity in modelling the system.

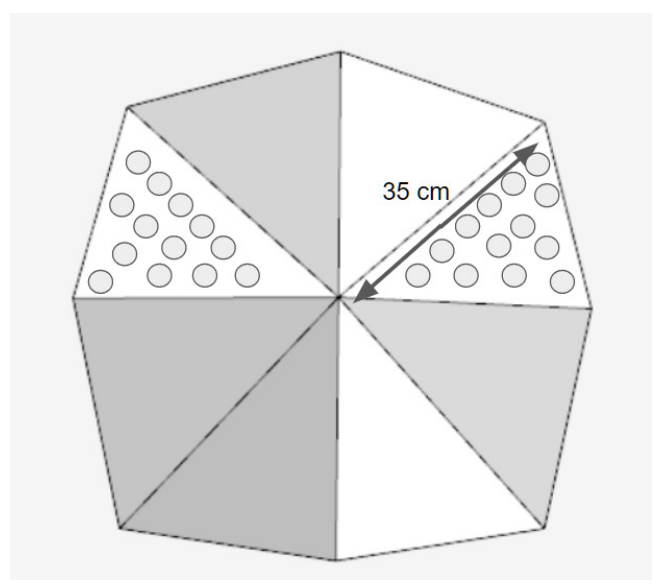

Figure 3.4: Position of the solar cells on the facade.

The solar cells are embedded on two surfaces of the facade as shown in Figure 3.4. Each side of this equilateral triangle is $35 \mathrm{~cm}$ long, making the total area of both the surfaces on a flap to be $0.106 \mathrm{~m}^{2}$. This means that a single block of the facade, which covers one window and consists of 15 flaps, will have solar panels that cover in an area of $1.6 \mathrm{~m}^{2}$. Putting this in perspective, a building like EWI in the Delft University of Technology can accommodate 20 such blocks on one side of the building, on each floor. The building has 23 floors, which brings the total area covered by solar cells on one side of the building to nearly $750 \mathrm{~m}^{2}$. 


\subsection{Conceptual Model Overview}

The system consists of various components, both hardware and software as illustrated in Figure 3.5. Sensors on the facade read out illuminance values periodically. Based on the sensor readings, an algorithm computes the energy the solar panels are receiving. A certain optimized value of the tilt angle $\theta_{\text {fos }}$ is computed using user preferences and a servo motor moves the block to that particular value of tilt. In case of sudden disruptions in light levels indoors that maybe caused due to cloud movements, the algorithm checks the values of deadband and delay time, as mentioned in the previous chapter and increases the levels of indoor lighting accordingly. The harvested energy is stored in a battery pack to enable the servo motors to run independently. All the excess energy is fed back into the grid with a meter keeping track of the energy surplus.

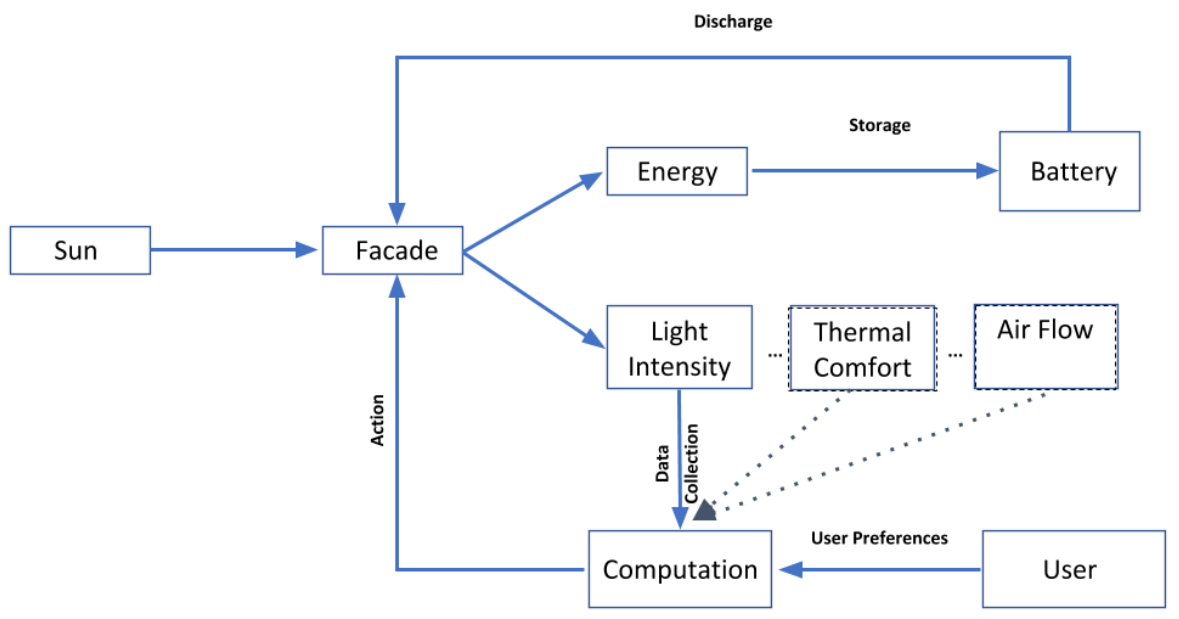

Figure 3.5: Block diagram of the conceptual FOS.

The vision of the concept includes not just the optimization of user comfort and energy, but also the simultaneous optimization of various parameters like thermal comfort, ventilation, humidity, etc., as shown in Figure 3.5 with dotted lines. Therefore, the optimization that the algorithm has to perform is a multi-objective optimization, which simultaneously optimizes multiple parameters. However, this work will only address the bi-objective optimization of user comfort and energy harvested. 


\subsection{High-level Workflow}

Once the model overview was envisioned, the next step was to define a highlevel working specification of the system. Figure 3.6 shows this workflow step-by-step. The system contains three models: Daylight, Solar Energy and User Comfort. The concept can have more models, depending on the number of parameters it aims to optimize. The models are pre-defined and calculate the various parameters defined within them, with the exception of the User Comfort Model. The Daylight Model contains the specifications on how the facade behaves with the particular indoor space with time and with varying weather conditions. The Solar Energy model calculates the energy that can be harvested from the facade at various tilt angles during the day. The User Comfort Model contains specifications with which the other two models function, in other words, it acts as a constraint on the working of the other two models.

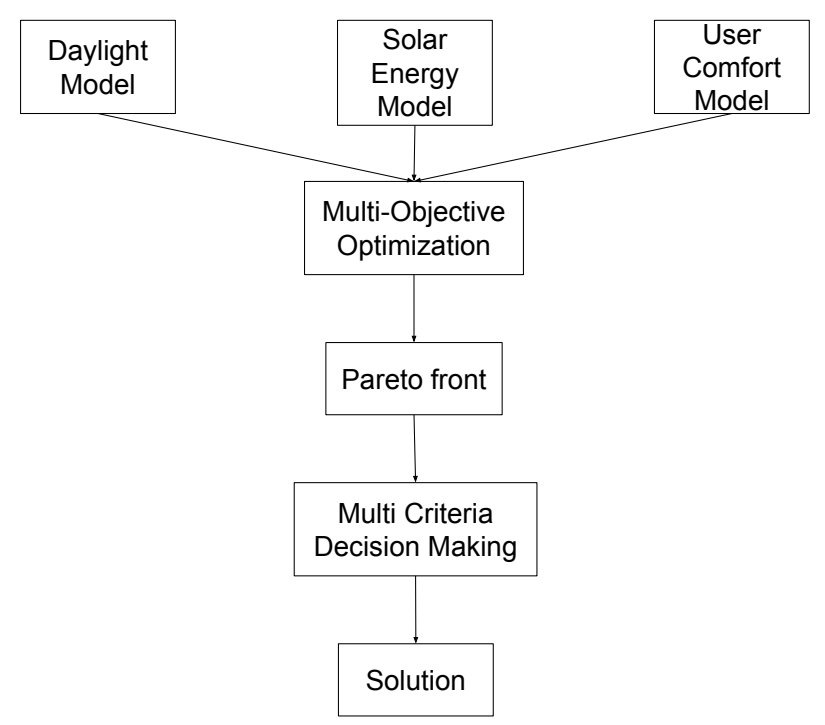

Figure 3.6: Flow diagram with high-level workflow of FOS.

These models acts as inputs to a multi-objective optimization problem, which is an optimization problem that requires more than one objective function to be optimized simultaneously. In such a scenario, there exists no single solution that simultaneously optimizes each objective. Hence, the objective functions are classified as conflicting, and there exists an infinite number of Pareto optimal solutions. The set of all Pareto optimal solutions is termed as the Pareto front. The goal in a majority of such optimization problems is to get as close as possible to this Pareto front. 
Multi-objective optimization problems have been studied for a long time using diverse techniques, depending on the nature of the underlying optimization problem. One approach for solving a one-dimensional function consists of taking weighted sum of each of the variables. Each set of these coefficients will lead to an optimal solution, depending on the weights. There are also other popular techniques like those based on a heuristic search, mostly involving genetic algorithms [24].

Translating these concepts to our problem, at every instant, the system looks to find an optimized tilt angle. Supposing this tilt angle determines the amount of light, air circulation and temperature inside the building, and the amount of energy harvesting from the facade, the tilt angle has to be chosen in a way that it satisfies the requirements of the user in all these parameters. In fact, it should not just satisfy the user, it has to optimize them all simultaneously. On performing design space exploration, all the requirements can be translated as functions. The graph of the feasible region for the constraints is called the design space for the problem. Evaluating the corner points of all the objectives gives rise to many line segments between the optimal points, representing possible solutions. The design space can then be translated into criterion space, which is achieved by converting the axes from variables to the objective functions. Upon analysis, an edge of the criterion space can be identified, on which each of the points represent an optimal solution. This is called the pareto front. Supposing both the axes were functions of energy and comfort, by moving along this pareto front, you could maximize energy at the cost of comfort or maximize comfort at the cost of energy, but you can't improve both at once.

There are also a number of multi-criteria decision making methods that could be applied to a Pareto front to find a solution. Methods such as weighted sum model, goal attainment, etc. exist but has to be chosen carefully according to the problem being solved. For the implementation of iFOS, we will not be using any multi-objective optimization as it is translated into a simple optimization problem. But these concepts are explained because the conceptual system would need a framework to identify the optimal solution. 


\section{Chapter 4}

\section{Discovering Light Dynamics and Energy Characteristics}

The interaction between light and the environment is fundamental to any system that looks to optimize lighting conditions. Light is a form of electromagnetic radiation, with the visible light constituting only a small fraction of the electromagnetic spectrum. Understanding the dynamics of light in the presence of a facade requires exhaustive learning of how light behaves around the specific geometry, also taking into consideration the geographical location, orientation and behaviour over time of the facade.

Illuminance level over a period of time at different tilt angles of the facade would help formulate a mathematical model capturing how the facade distributes this light inside the room. When extended to different times of the year and sky conditions, a database can be formulated that can show the behaviour of a facade. To test the facade and log data to accurately model the system, real world data is the best option. For the proposed location, a facade system designed and laser cut with high-grade cardboard would provide sufficient structural strength and aesthetic appeal to hold solar cells and control light entering the room. Servo motors attached to a gear system would control the flaps of the facade. A budget was made and it was deemed to be too expensive to be fabricated at such an early period of this research work. So, a small-scale model with simple hand controlled flaps was built. This model enabled the logging of light levels indoor for varying angles of tilt of the flaps. Since such a model would not capture the behaviour of a facade covering the entire window, it was decided that advanced simulations using ray-trace methods would be used to gather illuminance data. 
This research work relies on data recorded from advanced simulations to model the proposed facade optimization system. The goal of this learning process is to understand how light varies as the day progresses under varying levels of tilt of the facade. This can also be extended to various times of the year to see how it varies as the Earth is tilted on its axis and hence the path the sun takes in the sky varies through the year. It is critical to learn the characteristics of the facade as it helps bring out trends in the way the facade behaves, enabling a better modelling of the system.

\subsection{Facade Test-Bed}

To understand the correlation between the data generated by the simulations and the real-world scenario, a small, minimal test-bed was setup in an office space in the Faculty of Electrical Engineering, Mathematics and Computer Science at the Delft University of Technology with dimensions of $5.9 m \times 3.7 m \times 3.2 \mathrm{~m}$. A window is present in the room, measuring $3.6 \mathrm{~m} \times 2.4 \mathrm{~m}$

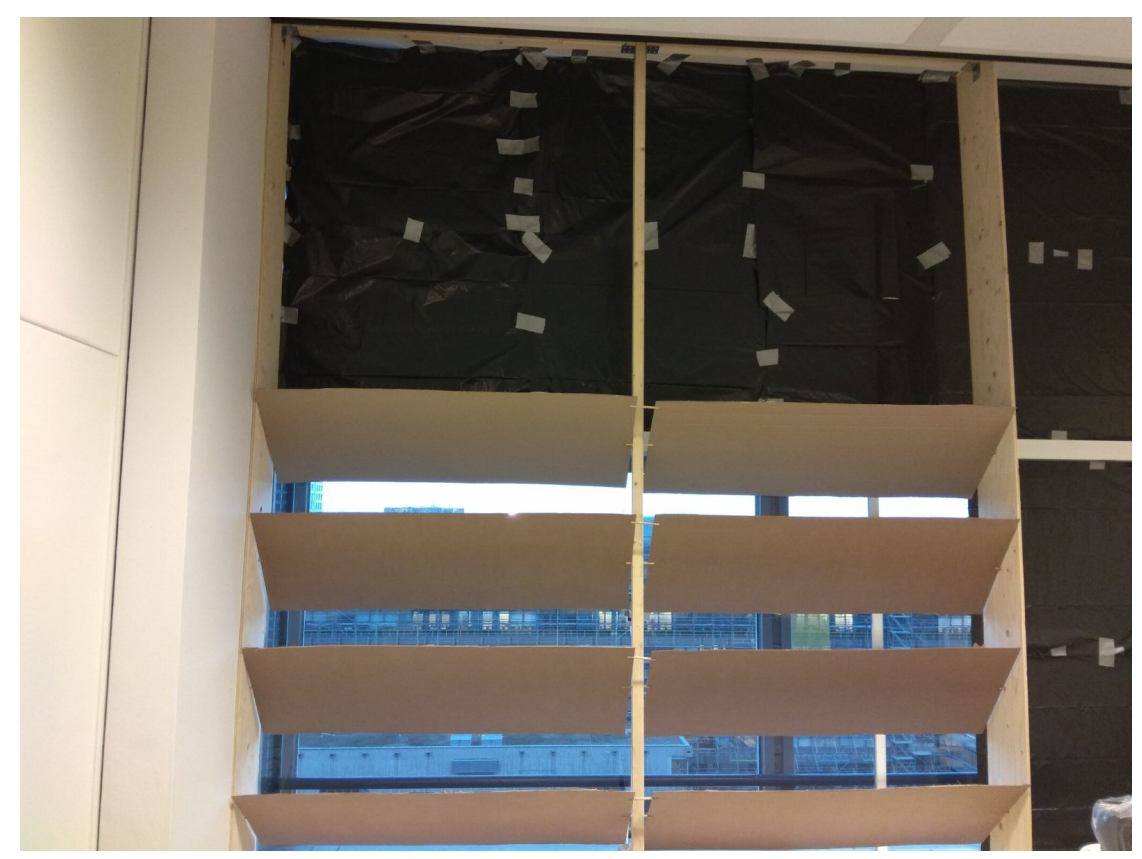

Figure 4.1: Small-scale test facade prototype.

at a height of $0.8 \mathrm{~m}$ from the floor. A facade system was made to cover a quarter of this window with a wooden frame and cardboard cut outs as flaps 
as shown in Figure 4.1. The system consisted of four flaps whose tilt angle could be adjusted from $0^{\circ}$ to $70^{\circ}$ with respect to the ground. Three sensing locations were identified to log illuminance values: a sensor on the window, facing outwards to measure the light levels outside, denoted as $S_{\text {out }}$; a sensor, facing the ceiling at a distance of $2 \mathrm{~m}$ from the window and a height of $0.8 \mathrm{~m}$ from the ground denoted as $S_{2 m}$; and a another sensor at a distance of $3 \mathrm{~m}$ from the window with the exact same specifications as the previous sensor, denoted as $S_{3 m}$. Every 30 minutes, illuminances values were collected from these three sensors for tilt angles varying in steps of $10^{\circ}$ from $0^{\circ}$, which is fully open to $70^{\circ}$, which is closed. Also, two sets of data were collected: one with only daylight and another with daylight and artificial lights turned on.

The goal of this experiment was to observe the behaviour of light and understand how factors like distance from the window and presence of artificial lighting affect the distribution of light indoors in the presence of a facade. To fully understand the behaviour, a full-scale facade, designed and engineered to maximize the energy gains and optimally control the light entering the room would have to be fabricated and installed.

\subsection{Simulating the Environment}

In order to simulate the environment, an environment is to be defined first. For this purpose, a computer-aided design (CAD) application software called Rhinoceros (abbreviated as Rhino) is used. The version used is the latest stable version, Rhinoceros 5 [25]. It's geometry is based on the mathematically oriented NURBS (Non-uniform Rational Basis Spline) model that focuses on producing precise representation of curves and free-form surfaces.

To accurately build the environment, a room in Faculty of Electrical Engineering, Mathematics and Computer Science at the Delft University of Technology was measured out. The dimensions of the room were the same as explained in the previous subsection. A facade structure covering the entire window was built and the surface geometries were made dynamic with the help of a Rhinoceros plugin called Grasshopper [26]. Grasshopper is a visual programming language that runs within Rhino and is primarily used to build generative algorithms or create 3D geometry. It is used to give dynamics to the facade that enable it to fold into various angles and enable structures that can be used to analyze various physical parameters. Figure 4.2 shows a screenshot from the initial development of the simulation. The room was drawn out and the facade along with its dynamic components in Grasshopper was added. 


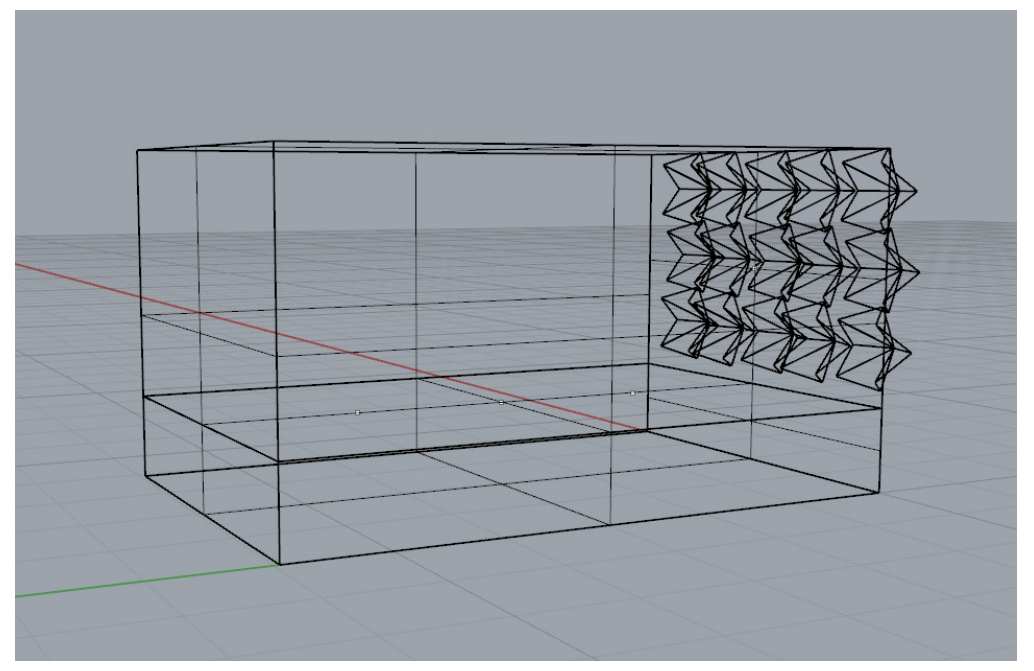

Figure 4.2: Test bed environment simulated in Rhinoceros 5 .

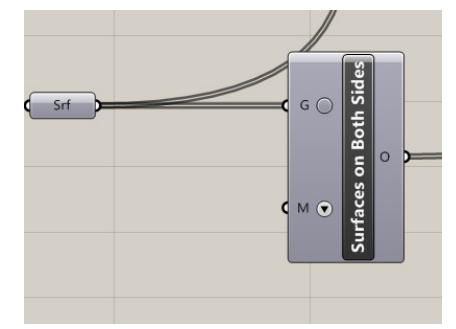

Figure 4.3: Material definition block in Grasshopper.

Surfaces defined on Rhino are assigned geometries, which can then be assigned a Material ID in Grasshopper. The options for this range from generic material types of various reflectances and glazing levels which can be chosen by the user. Figure 4.3 illustrates the block in Grasshopper that defines surfaces their materials with a drop-down menu $M$. This step is crucial in the lighting analysis as it enables the simulations to be as accurate as possible.

Figure 4.4 shows the Grasshopper definition of various surfaces in the simulation. Each of the surface blocks have three inputs: list (L), index (i) and wrap (W). The surface assigned a material serves as the input list $L$ and is given a unique index $i$. Wrap is used to wrap index to list bounds. Since we assign a unique index for every surface, this option is left blank. The resulting items are then clustered and serve as object input surfaces to the daylighting analysis. So far, the room and the facade have been simulated and the final, crucial element to be simulated is the Sun. 


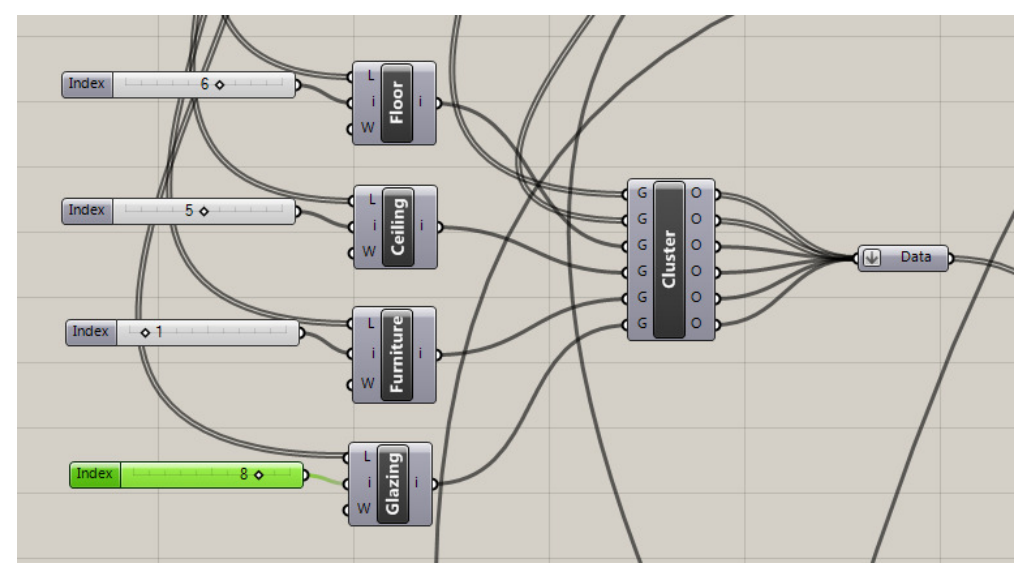

Figure 4.4: Indexing and clustering of different surfaces.

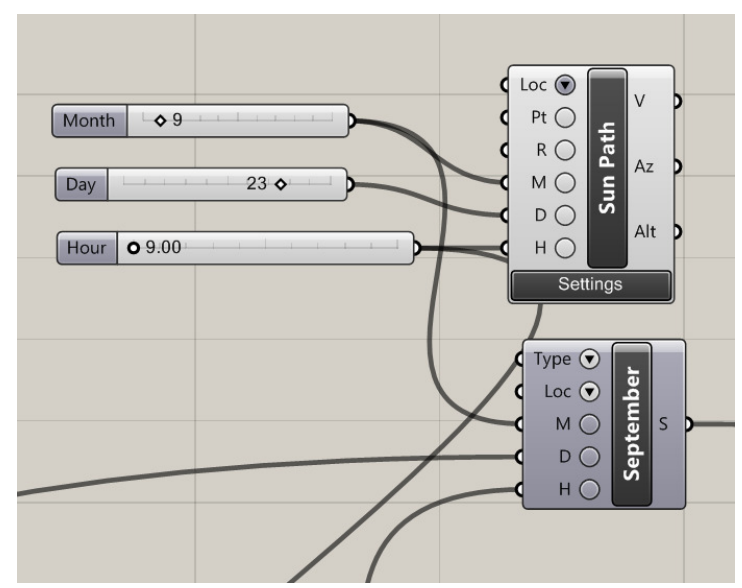

Figure 4.5: Sun Path component in Grasshopper.

As the Earth rotates around the Sun on its annual cycle, it is tilted at an angle on its vertical axis. This has a direct impact on how the sun's rays strike different locations on Earth. Depending on the time of the year, the sun's path in the sky in relation to the horizon varies from low to high. However, this variation is highly predictable and the concept of passive solar design uses these predictable movements of the sun to best utilize its energy within the building's design. In Rhino, the sun can be simulated using a component in Grasshopper called Sun Path. This component creates a 3D sun-path and also outputs sun vectors that can be used for sunlight analysis or other shading analysis. A component diagram is shown in Figure 4.5, clearly showing the inputs to a Sun Path block. The month, day and hour can be defined to plot an hourly sun-path diagram. The location of the simulation is to be defined through the Loc drop-down list, which primarily requires TMY (Typical Meteorological Year) files to be loaded. In this case, 
Amsterdam, The Netherlands was the best match geographically and was used as the location for the simulations. The important output here is the solar vector $V$ which tells us the location of the Sun in a 3D plane, as shown in Figure 4.6. The orientation was set to South to be able to catch a 180 degree field of view as the sun moves across the sky. If the location was in the southern hemisphere, then it had to be set to North to be able to catch this 180 degree field of view. This is done in order to understand the dynamics of the facade from all possible incidence angles of the sun's rays.

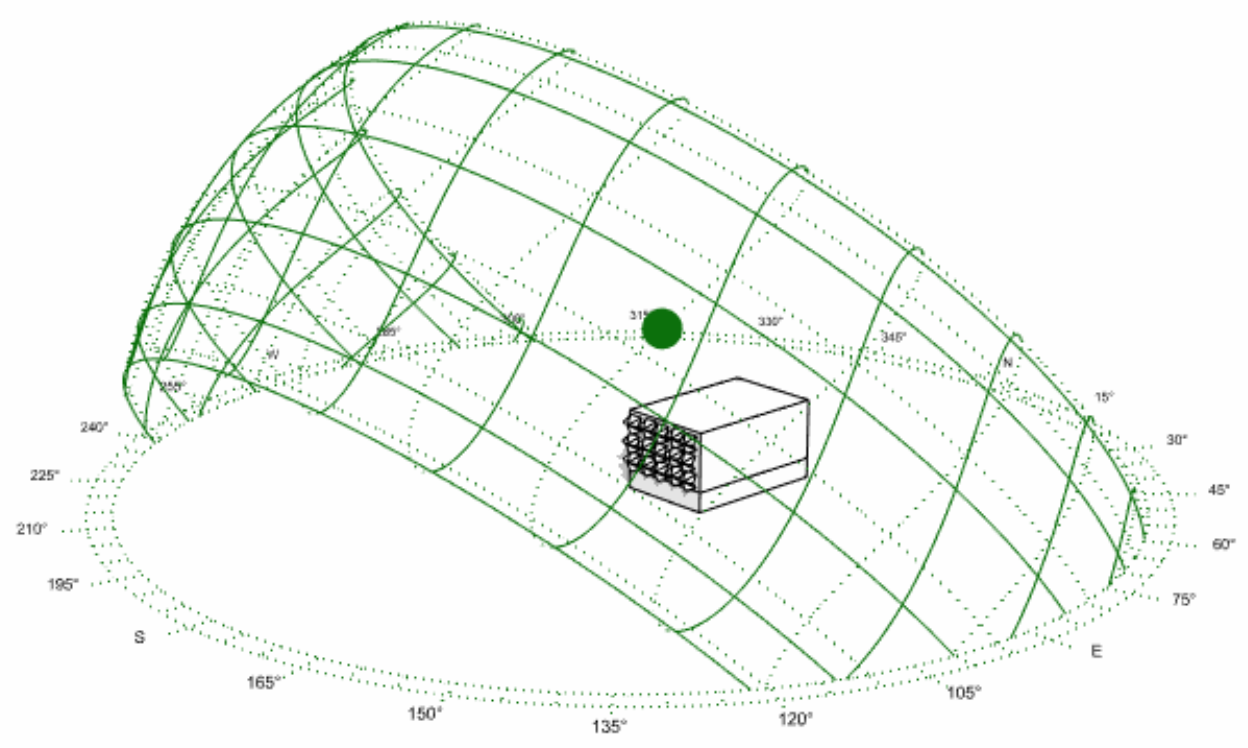

Figure 4.6: The simulated path of the Sun across the facade using Sun Path. 


\subsection{Daylighting Analysis}

The next major step in the learning process was to perform daylighting analysis on the simulated test bed. For this, a specialized, highly optimized daylighting and energy modelling plug-in for Rhino called DIVA was used. The plug-in was initially developed at Harvard University and is now developed and distributed by Solemma LLC [27]. Within the DIVA framework, the type of simulations used are called Climate-Based Metrics and Daylight Illumination. It uses recorded climate data to simulate the sun and sky conditions for various types of simulations like Daylight Autonomy, Daylight Availability and Useful Daylight Illuminance. It is also important to note that these simulations are annual calculations and can calculate values for any day of the year. They use calculations engines like RADIANCE [28] and DaySim [29] for metrics and use techniques like ray tracing to perform all the lighting calculations.

The room in the simulation is divided into defined grids and lighting inside is calculated for each of these grids. The simulation allows the user to set pre-defined locations where the illuminance is to be measured by setting up nodes. Figure 4.7 shows the location of the sensor nodes in the simulation by highlighting them. Three equidistant sensors, namely $S_{1}, S_{2}$ and $S_{3}$ are placed $0.8 \mathrm{~m}$ from the ground and one sensor is placed right on the window, behind the facade, termed as $S_{w i n}$.

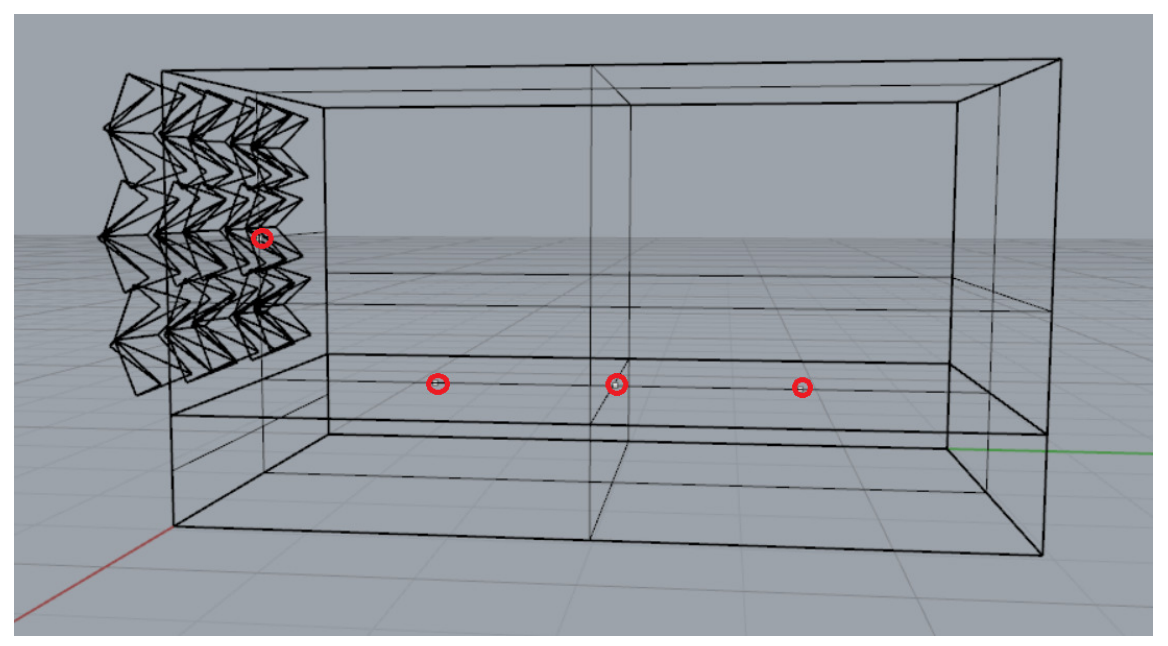

Figure 4.7: Illustration of the positioning of sensors in the simulation.. 


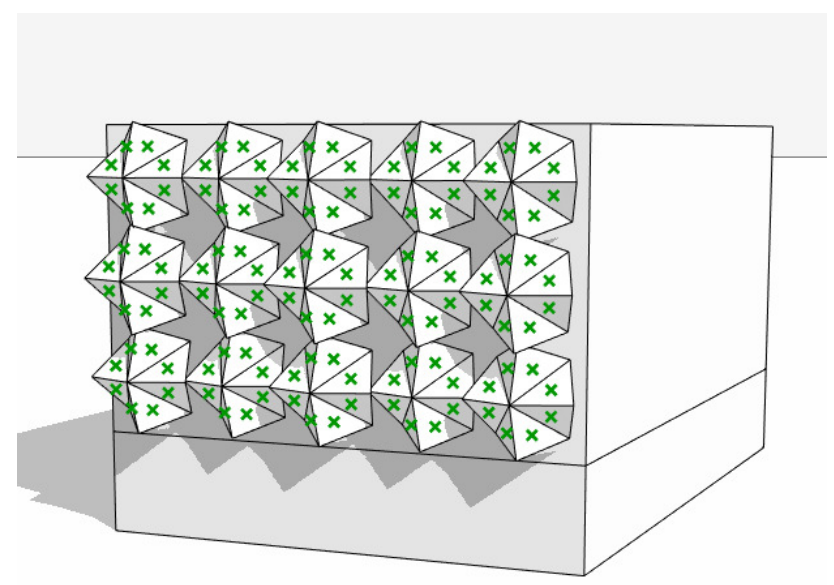

Figure 4.8: Data points on the surface of the facade.

The amount of illumination on the surface of the facade, termed as $S_{\text {out }}$ is measured by calculating the average value of light falling on multiple points on the facade, as illustrated in Figure 4.8. The simulations works by calculating the irradiance values on the surfaces defined by dividing them into grids of user-defined sizes as illustrated in Figure 4.9. It shows the grids with their individual illuminance values, to be seen as a top-view of the ceiling. The reflectance of the surfaces are taken into account and the software then uses ray tracing methods to calculate the illuminance values at the sensor nodes.

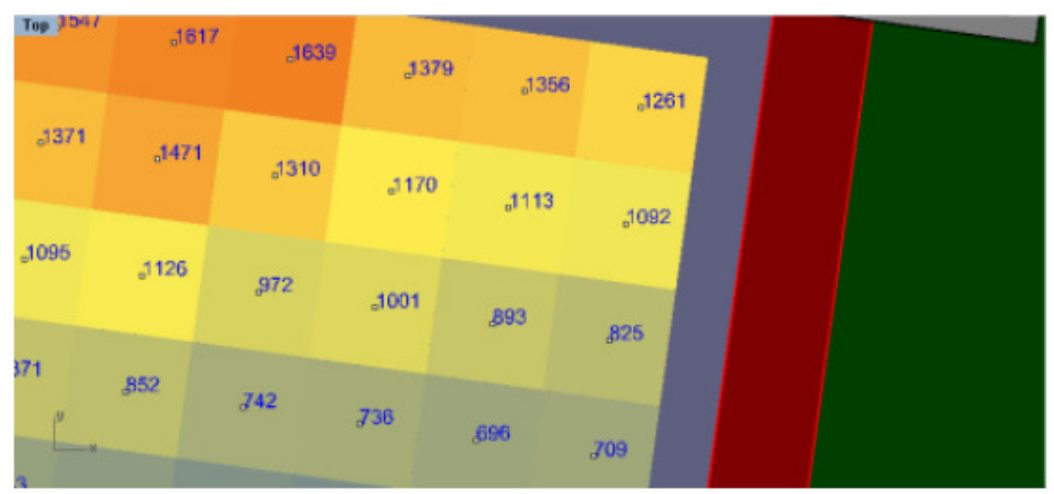

Figure 4.9: View of the grids computed inside the room on running Daylighting Analysis. 
The simulations were performed from 09:00 hrs in the morning till 17:00 hrs in the evening to maintain uniformity as the simulations don't work the moment the sun goes below the horizon. Hence, we have 100 illuminance values over the course of the day, roughly one sensor reading every 5 minutes. The simulations were also performed for three different sky conditions: Clear Sky, Intermediate Sky and Overcast Sky. For a particular sky condition, the simulation over the course of the defined time takes roughly 2 hours to complete. This meant that a brute-force approach to compute for the entire year would be highly time-consuming.

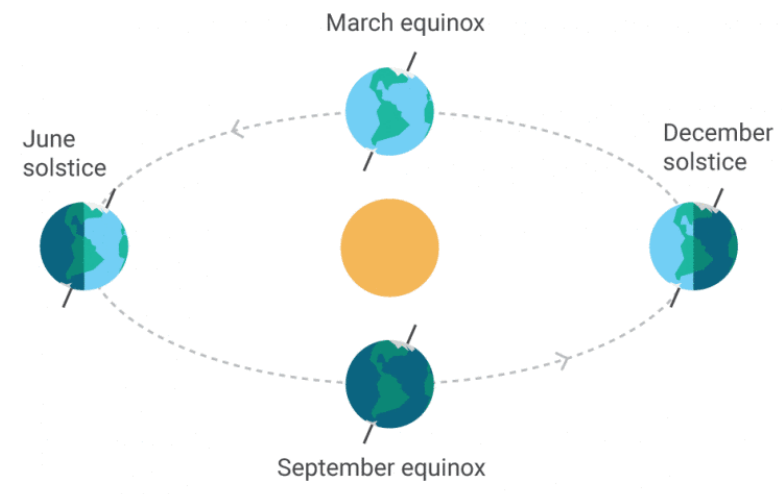

Figure 4.10: Illustration of the equinoxes and solstices [30].

The Earth's axis of rotation is tilted from its orbital plane around the sun, as a result of which the orientation of the Earth's axis to the Sun is constantly changing as it revolves around the Sun. Along with this change in the orientation, the amount of sunlight falling on its surface also changes, causing a seasonal variation in the amount of sunlight received, varying with latitude also. This gives rise to many extreme days of the year, with the maximum amount of sunlight and minimum amount of sunlight received. Hence, it was concluded that to understand the dynamics of how the varying position of the sun in the sky through the year affects lighting conditions indoor, the four corner days of the year would be simulated, namely the equinoxes and the solstices as illustrated in Figure 4.10. Each of these days would be simulated with the three different sky conditions enabling a dataset that contains five sensor readings, three different sky conditions and eight different orientations of the facade, from $5^{\circ}$, which is fully open to $75^{\circ}$, which is fully closed. This results in 480 sets of illuminance data of 100 values each. 
To perform an energy optimization simultaneously, the corresponding solar energy data also had to be computed and this brought us to the next step in the process.

\subsection{Solar Energy Calculations}

The characteristics of the solar cells embedded on the facade had to be studied and this was possible through purpose-built MATLAB functions. The position of the sun can be found by using the Solar Postion Algorithm [23], as explained in Chapter 2. Following this, we use the purpose-built MATLAB function to calculate the amount of energy falling on the facade. The steps taken to calculate this value is explained below:

1: Input: Surface Tilt, Surface Azimuth, Solar Zenith, Solar Elevation, Solar Azimuth, Direct Irradiance, Diffuse Irradiance.

2: Calculate the Direct Normal Irradiance (DNI) by dividing the direct irradiance by $\sin$ (Solar Elevation).

3: Calculate Global Horizontal Irradiance (GHI) by adding Direct Irradiance and Diffuse Irradiance.

4: Calculate the angle of incidence (AOI) for every tilt angle of the facade from the set of possible tilt angles using the MATLAB function getaoi.

5: Calculate the total energy harvested with the MATLAB function irr-poa.

6: Output: Energy harvested $\left(W / m^{2}\right)$

Algorithm 2: Solar Energy Calculator

The dataset containing the direct and diffuse components of solar radiation were obtained from the Koninklijk Nederlands Meteorologisch Instituut (KNMI) [31]. Historical data was also extensively used from the PANGAEA database of KNMI [32]. In The Netherlands, KNMI has numerous weather stations but only one of them, Cabauw, measures the different components like direct and diffuse radiation. The others only measure the global radiation and hence all the data of solar radiation in its components come from Cabauw. It is located about 60 kilometers east of Delft, in the province of Utrecht. Therefore, the values used for calculations may or may not be representative of the sky conditions in Delft. KNMI datasets contain hourly-averaged values of the diffuse and direct irradiances. 
The facade test-bed was also used to log data at various tilt angles with a solar panel measuring $10 \mathrm{~cm} \times 8 \mathrm{~cm}$ with about $15 \%$ conversion efficiency. Measurements were taken after the solar cell was mounted onto the facade and the tilt angle was varied in steps of $10^{\circ}$ from $0^{\circ}$ to $70^{\circ}$. The corresponding values of voltages generated by the solar cells were measured with the help of a multimeter.

To calculate the total amount of electricity generated by a photovoltaic system, the following formula can be used:

$$
E=A \times r \times H \times P R
$$

where,

$E$ is the amount of electricity generated in $\mathrm{kWh}$.

$A$ is the total area of solar panels in $m^{2}$.

$r$ is the solar panel efficiency.

$H$ is the average annual solar radiation on the panel, not considering shading effects.

$P R$ is the performance ratio, varying between 0.5 to 0.9 , depending on various factors. 


\section{Chapter 5}

\section{Optimization Formulation and Algorithm}

This chapter firstly introduces the concept of multi-objective optimization. The first one deals with formulating the optimization problem. The case will be looked first as a problem for a single block and then generalized into the case for a whole building. Following this, arguments are made for the optimization formulation and an algorithm is proposed that best suits the arguments.

\subsection{Problem Definition}

The goal of the optimization problem at hand is to maximize user comfort in indoor spaces while simultaneously optimizing energy harvested from solar panels embedded on the facade. User comfort can be translated into many different parameters, some of which are thermal comfort, visual comfort, acoustic comfort and air quality. There have been numerous studies that developed methods to optimize these parameters either separately $[33,34,35,36]$ or simultaneously $[37,38,39]$. The scope of this research work will be to propose an optimization between visual user comfort and energy harvested. Hence, user comfort will be translated into amicable lighting conditions indoor.

Sarkar et al. devised a method to collect and interpret information regarding how occupants preferred various lighting levels [40]. They represented the lighting preference as a beta function after a study that involved multiple users. This study took place in the same geographical location of Delft, The Netherlands as this work and hence is considered an important set point in 
this optimization. As defined by their work, illuminance levels ranging from 300 lux to 700 lux was within a range they called preferable. A light level of 500 lux formed the peak of this beta curve and was hence found to be the most desirable light level. It was also found that the best way to make changes in illuminance levels was in steps of 25 lux.

Based on these above mentioned findings, the preferred light level to be maintained indoors is set in the range [300,700] lux with 500 lux being the best illuminance level. Since our goal is to maximize user comfort, we further define a three-level user comfort setting:

- High: $[450,550]$ lux

- Medium: $[400,600]$ lux

- Low: $[300,700]$ lux

- No Occupancy: 1 lux, i.e. zero weight to comfort.

The level of comfort will be set by the user with the knowledge that a lesser user comfort may translate into higher energy gains. If the system detects that the user is not present in the room, then it automatically turns itself into the low comfort mode after waiting for a certain buffer time.

\subsection{Problem Formulation}

The goal of the optimization problem is to maximize user comfort $c$ while maximizing energy harvested, denoted by the plane of array irradiance $E_{\mathrm{POA}}$, which is the total irradiance on the facade. Thus the problem can be formulated as:

$$
\text { Maximize }\left\{\begin{array}{l}
c=f\left(\theta_{\text {fos }}\right) \\
e_{\text {total }}=g\left(\theta_{\text {fos }}\right)
\end{array}\right.
$$

both of which are functions of the tilt angle $\theta_{\text {fos }}$, subject to the constraints,

$$
5^{\circ}<\theta_{\text {fos }}<75^{\circ}
$$

Chapter 3 explained that FOS will consist of a block for every room, consisting of 15 flaps each that will be controlled as one entity.

For generalizing and formulating this, we assume that every flap is capable of setting its own tilt angle. Let us assume that there are $n$ flaps in 


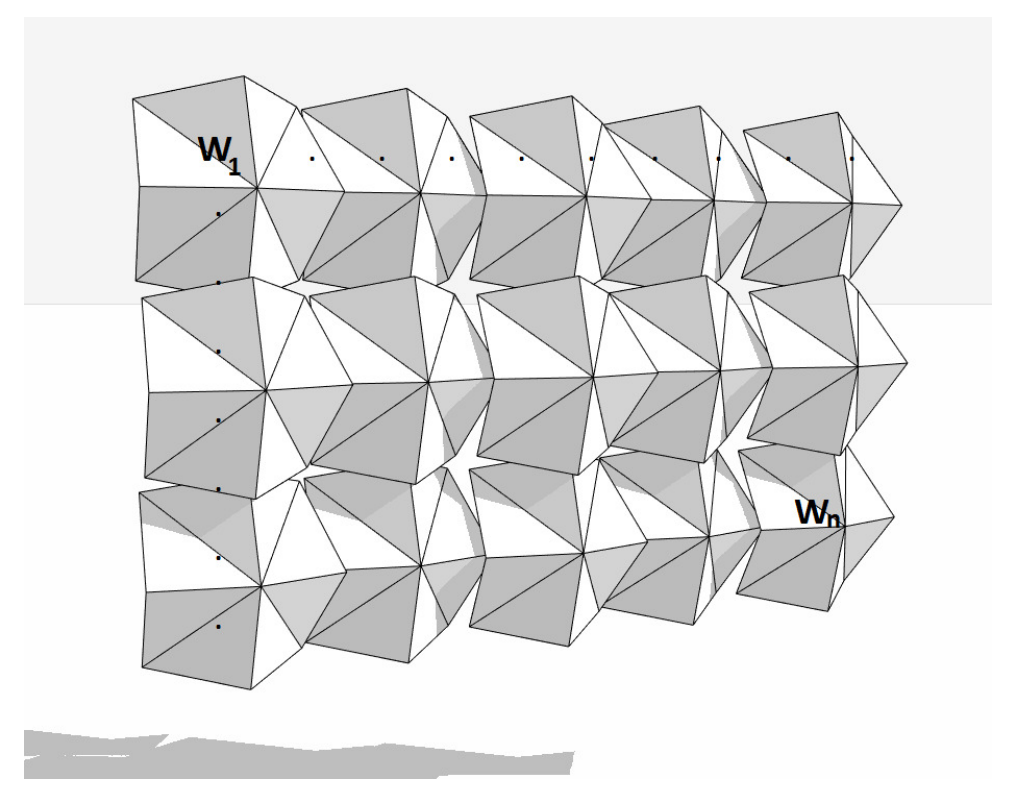

Figure 5.1: Illustration of individually moving flaps.

a block sitting on a window, denoted as $w_{i}$ for the $i^{t h}$ flap as illustrated in Figure 5.1 and each of them is capable of having their own tilt angle $\theta_{\text {fos }}^{i}$. The sensors just outside and inside the flap $w_{i}$ are represented as $S_{\text {out }}^{i}$ and $S_{i n}^{i}$ respectively. A relation has to be devised between these two sensors that take into account the dynamics of the behaviour of the flaps. Their values can be related using the equation:

$$
S_{\text {in }}^{i}=a_{i} S_{\text {out }}^{i}\left(\frac{\theta_{\mathrm{fos}}^{i}}{\theta_{\max }}\right)+b_{i}
$$

where $\theta_{\text {fos }}^{i}$ is the tilt angle of the flap $w_{i}, \theta_{\max }$ is the maximum tilt angle, which in this case is set by design choices at $75^{\circ}, a_{i}$ and $b_{i}$ are the regression parameters that need to be learned from the model. For every flap, this can either be the same value or different, depending on the mode of data collection.

Now, for any location $j$ inside the room, the total illuminance at that point $S_{i n}^{j}$ is represented as:

$$
S_{i n}^{j}=\sum_{i=1}^{n}\left(c_{i j} S_{i n}^{i}+d_{i j}\right)
$$

where $c_{i j}$ and $d_{i j}$ are parameters that need to be learnt. 
A few key challenges in learning that arise out of this definition are:

- Given that there are $n$ flaps and $m$ values of tilt angle $\theta_{\text {fos }}^{i}$, there could be $n \times m$ combinations for the configuration of the flaps in a block. However, this can be simplified if all the $a_{i}$ 's and $b_{i}$ 's take the same value, i.e. each block is guided by the same parameter values.

- Given that there $z$ locations inside the room where the illuminance is to be maintained, $n \times z$ variables, i.e. values of $c_{i j}$ 's and $d_{i j}$ 's need to be learnt assuming that the values of $a_{i}$ and $b_{i}$ is the same for all the flaps.

Now, further defining the optimization problem, our goal is to maximize the user comfort while maximizing the energy harvested from the solar cells on the facade. The user comfort is translated into a variable $c$ which is defined in terms of the light levels indoors. We had previously explained that a light level of 500 lux was found to provide user's maximum comfort. Hence, we define $l_{o p t}$ as the value of light that gives maximum user comfort.

We also define a value of the lighting condition indoor $l_{i n}$, which is the daylight entering the room through the facade, as a function of $f\left(\theta_{\text {fos }}\right)$. The energy harvested by the solar cells depend on the sun's location and the tilt angle of the facade $\theta_{\text {fos }}$. The individual contribution of every flap is added together to find the total energy harvested by the facade. Hence, this can be represented as a function of the sun's location and the tilt angle of the facade.

The problem is then formulated as:

$$
\text { Maximize }\left\{\begin{aligned}
c & =\frac{l_{\text {opt }}}{\left|l_{\text {opt }}-l_{\text {in }}\right|} \\
e_{\text {total }} & =\sum_{i=1}^{n} e_{i}\left(\theta_{A}, \theta_{Z}, \theta_{\text {fos }}^{i}\right)
\end{aligned}\right.
$$

where,

$$
\begin{gathered}
l_{\text {opt }}=500 \\
l_{\text {in }}=f\left(\theta_{\text {fos }}\right) \\
5^{\circ}<\theta_{\text {fos }}<75^{\circ}
\end{gathered}
$$

$\theta_{A}$ which is the solar azimuth and $\theta_{Z}$, the solar zenith together represent the sun's location. $\theta_{\text {fos }}^{i}$ is the tilt angle of the $i^{\text {th }}$ flap of the facade. 
Our goal is to maintain the light levels indoor as close as possible to this optimal level that ensures maximum comfort. But doing so results in lower levels of energy harvested from the solar cells. Here, we use the previously defined concept of having three levels of comfort to be chosen by the user, which further defines the range within which $L_{i n}$ will optimize the illuminance levels.

Suppose we have $n$ flaps in the facade of a building, each with $y$ possible tilt angles, this makes our search space atleast of the magnitude of $n \times y$. Therefore, finding a solution to the optimization problem is not trivial. Hence, an algorithm is defined in the following subsection that finds a solution to the optimization problem.

\subsection{FOS Controller}

The goal of the facade controller is to ensure that the tilt angle is set to the optimized angle, taking into consideration the amount of light let in and the energy harvested from the solar cells embedded on it. In case the daylight is insufficient to light up the work plane, it also sends the values of corresponding artificial lighting levels to the lights in the room. This is achieved because of the theory of radiosity, wherein if we know the light falling on the work plane $l_{w}$ and the user-defined preference level $l_{u}$, then the light deficiency $l_{d}$ is,

$$
l_{d}=l_{u}-l_{w}
$$

First, the algorithm computes the position of the sun in the sky by taking into account the time and day of the year. With this information, it is able to compute the amount of energy the solar cells can harvest. It also has information of the user preferences based on who the present occupants of the room are and it decides a common set-point of light level by taking the maximum of all the occupants' comfort values. Then, with all this available data, it derives a set of solutions of light levels and amount of energy harvested.

Following this, weights are iteratively added to both the objectives. From this derived set of solutions, the algorithm minimizes the light level within that particular comfort setting, i.e. if user chooses High comfort, it minimizes the light level in the set $[450,550]$ lux and chooses the value of $\theta_{\text {fos }}$ that satisfies the condition. Finally, the changes to the facade angle are brought about in small steps so that the user does not notice a sudden change in light levels or the movement of the facade. This step-by-step movement 
stops when the facade reaches its target tilt angle and this time period is termed as fade time. Another term is also designed into the algorithm called delay time. This is the amount of time the system waits before it acts on changing the light level or the tilt angle of the facade. Events such as moving clouds are identified by reading out the indoor sensors and looking for changes greater than 100 lux within 5 seconds or more. This is solved by increasing the indoor light in steps of 25 lux, which has been shown in [40] to be an optimal level of change.

Run Algorithm 1

Run Algorithm 2

Input: $\mathrm{e}\left(\theta_{\text {fos }}\right)$, Illuminance indoor $\left(l_{\text {in }}\right)$, where $l_{\text {in }}=\mathrm{f}\left(\theta_{\text {fos }}\right)$.

Output: Tilt angle $\left(\theta_{\text {fos }}^{\text {optimal }}\right)$

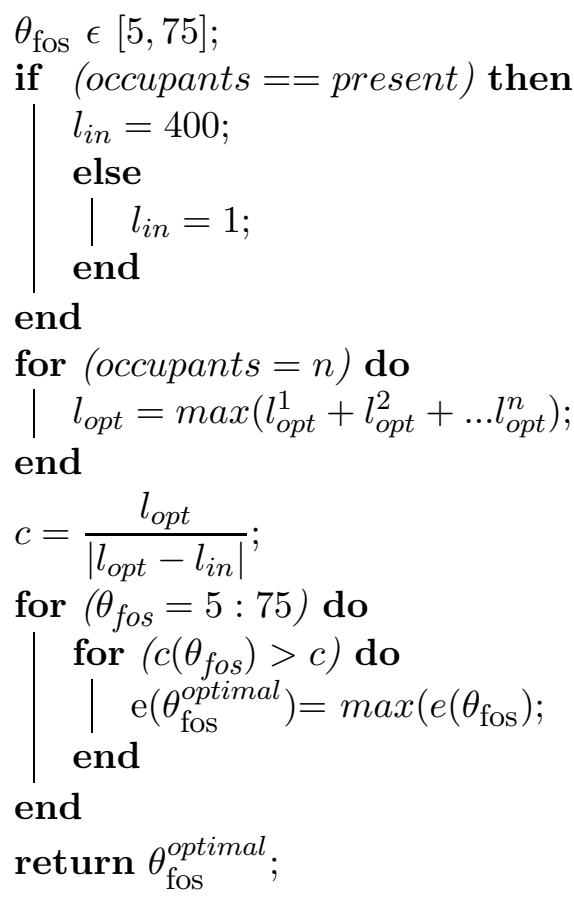

Algorithm 3: The proposed algorithm iFOS. 


\section{Chapter 6}

\section{Evaluation}

In the previous section, we explain the formulation of the optimization problem in detail, and an algorithm is proposed to solve the problem. Before we evaluate the proposed algorithm, a number of other evaluations and results are discussed as well. These are the outcome of a number of experiments performed and evaluation of the simulations over the course of this research work.

\subsection{Facade Test-Bed}

In section 4 , it is explained that a scale model of the facade is made to study the dynamics of light in the room. Since building a bigger facade model is not feasible, this work relied on advanced simulations to gather data in order to build the model.

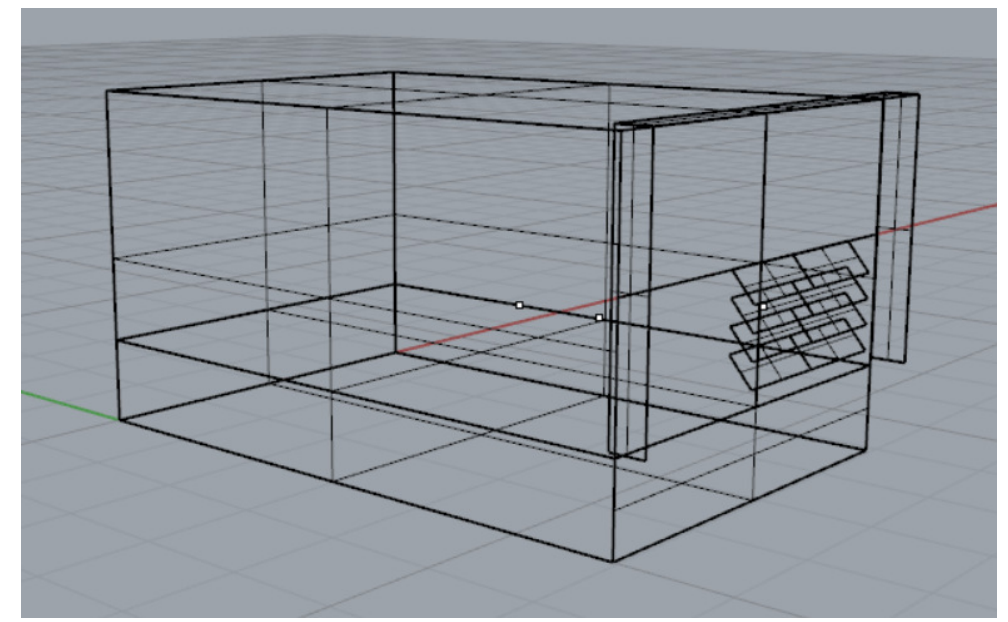

Figure 6.1: Simulated facade test-bed in Rhinoceros. 
However, it is indeed critical to evaluate the accuracy of the simulations to the real-world data. Hence, the facade test-bed is evaluated with the help of its corresponding simulation in Rhinoceros with DIVA plugin. This simulated environment is different from the one created for the conceptual facade. Figure 6.1 shows the test-bed environment simulated in Rhinoceros, where the facade can be tilted in angles varying from $0^{\circ}$ to $70^{\circ}$, measured from a horizontal plane. Two sensors were placed at a distance of $2 \mathrm{~m}$ and $3 \mathrm{~m}$ from the facade, at a height of $0.8 \mathrm{~m}$ from the ground. The path of the sun was simulated and a daylighting analysis was performed on the room. For this, various generic reflectance values were assigned to different surfaces like ceiling, floor, etc. for accurate results. Figure 6.2 illustrates the grid-view of the test-bed, clearly showing how the light reflects off surfaces.

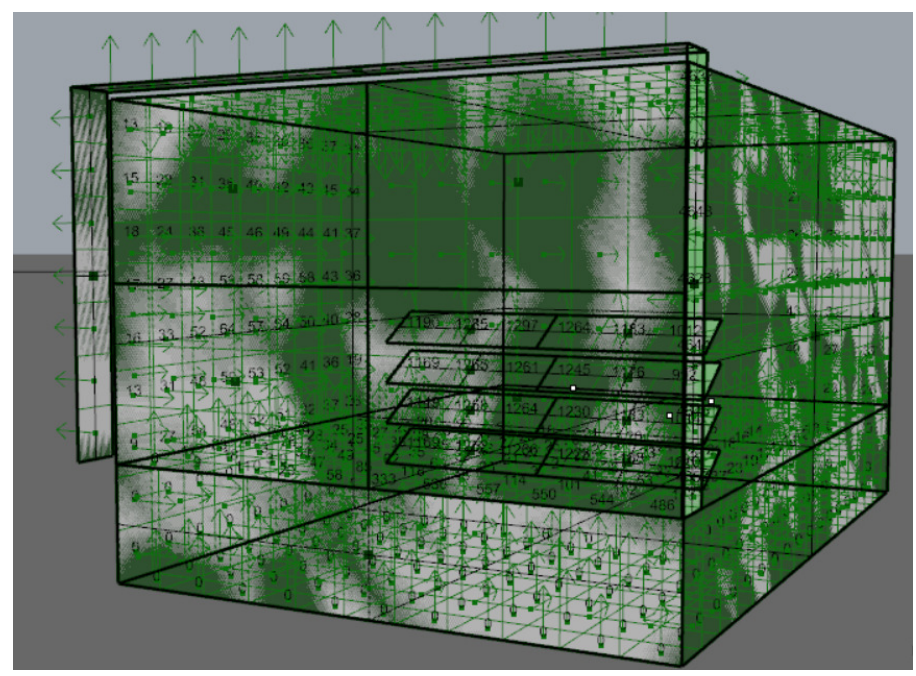

Figure 6.2: Grid-view of the simulated test-bed.

\section{Results}

Measurements are taken at intervals of 15 minutes, first at the window, followed by two locations at $2 \mathrm{~m}$ and $3 \mathrm{~m}$ from the facade. The experiment is carried out from $11 \mathrm{AM}$ till $5 \mathrm{PM}$ on the $10^{\text {th }}$ October, 2017. The tilt angle of the facade is incremented in steps of $10^{\circ}$, starting from $0^{\circ}$, which is fully open till $70^{\circ}$. The illuminance values are measured with the help of a lux meter, with the sensor facing the ceiling. This is done in order to capture the incoming illuminance onto the work plane. 


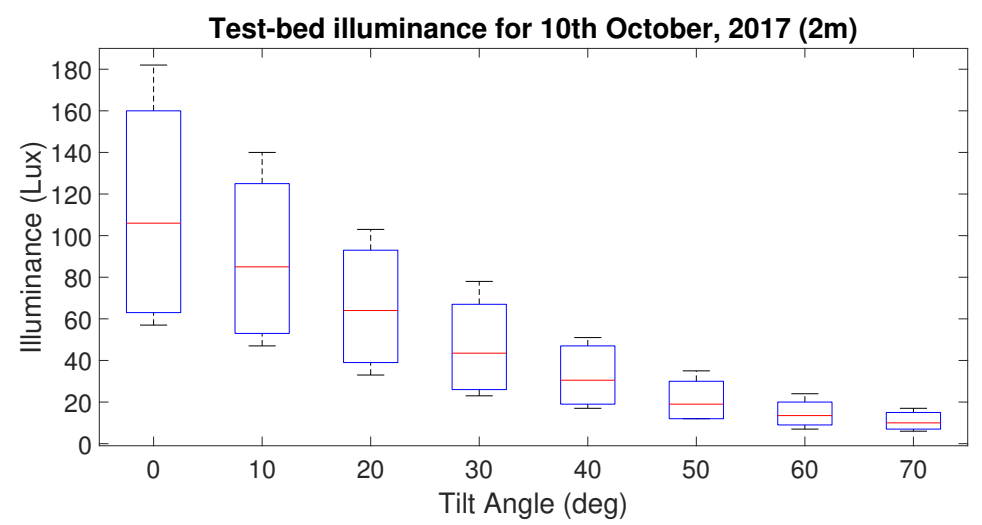

(a) Test-bed

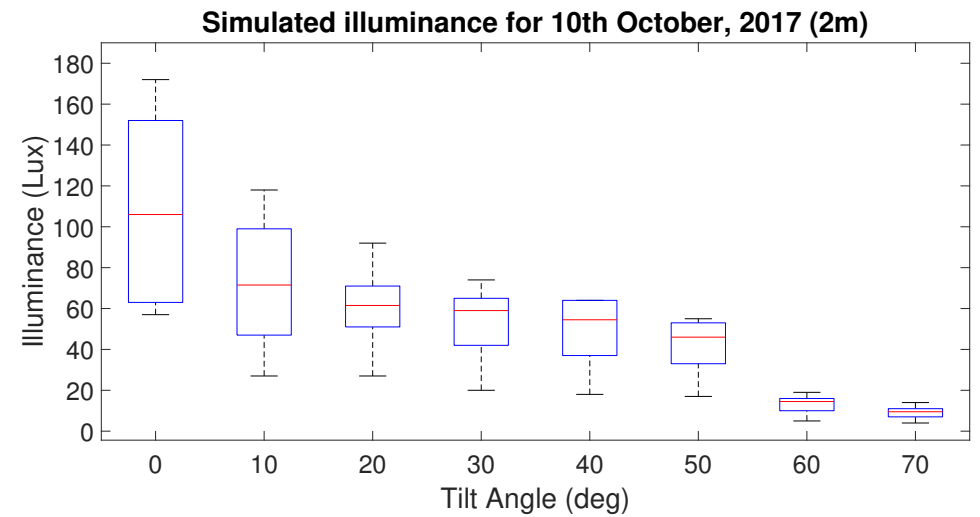

(b) Simulated

Figure 6.3: Comparison of the box plot of illuminance data for the sensor at $2 \mathrm{~m}$.

Figure 6.3 shows a box plot comparing the illuminance data from the testbed to that of the simulated test-bed. It can be seen that both the plots have a similar pattern in variation of illuminance levels with varying tilt angle along the day. It can also be inferred that the maximum and minimum illuminance values for various tilt angles are also almost identical.

The correlation between the real and simulated data is also computed with the MATLAB function fitlm in linear mode. An average R-squared value of 0.802 shows how closely the two sets of data are related in magnitude. Similar analysis is also carried for the sensor located at $3 \mathrm{~m}$ from the facade, as illustrated in Figure 6.4. An average R-squared value of 0.754 is observed for the two sets of data. 


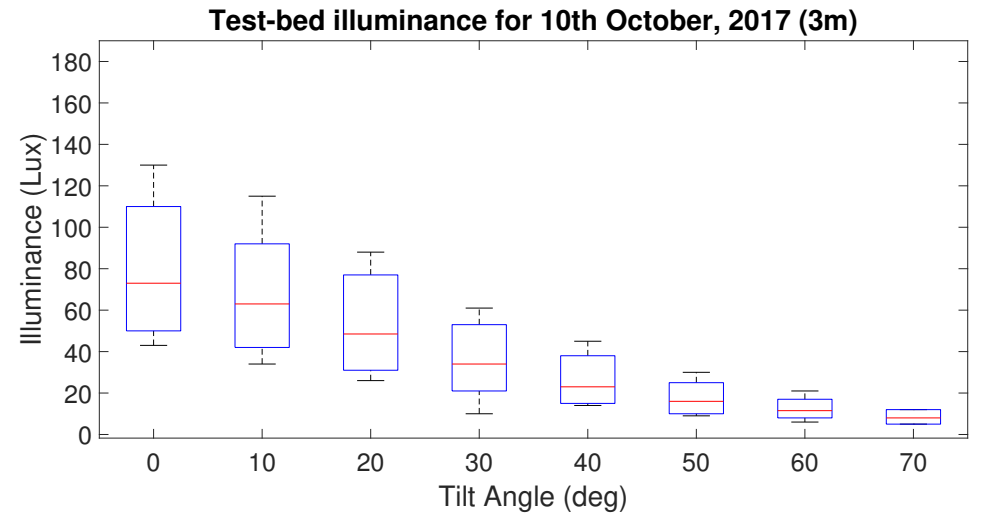

(a) Test-bed

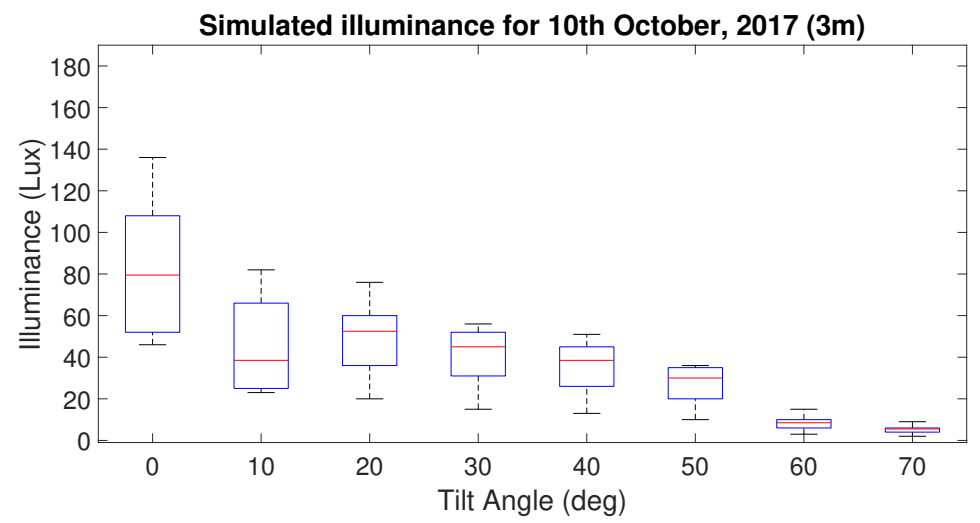

(b) Simulated

Figure 6.4: Comparison of the box plot of illuminance data for the sensor at $3 \mathrm{~m}$.

From these results, it can be concluded that the simulations performed with Rhinoceros and DIVA plugin offer a high level of accuracy to real-world scenarios and hence can be used to model an entire conceptual facade. 


\subsection{Simulations of the conceptual facade}

The previous section provides substantial evidence towards the accuracy of the simulations performed with Rhinoceros and DIVA in comparison to real-world scenarios. Hence, a large-scale system, for an entire window is modelled in Rhinoceros and this section evaluates the data from these simulations. For such a system, the best reference database is one which is simulated by brute-force for all possible days of the year with varying sky conditions. Since this is resource intensive, we could not perform these simulations for an entire year. However, it is clear from the data that it is possible to preserve the characteristics in the dynamics of light throughout the year by simulating the four extreme days of the year, both the equinoxes and the solstices. Hence, the simulated days are: 23rd June, 23rd September, 22nd December and 21st March.

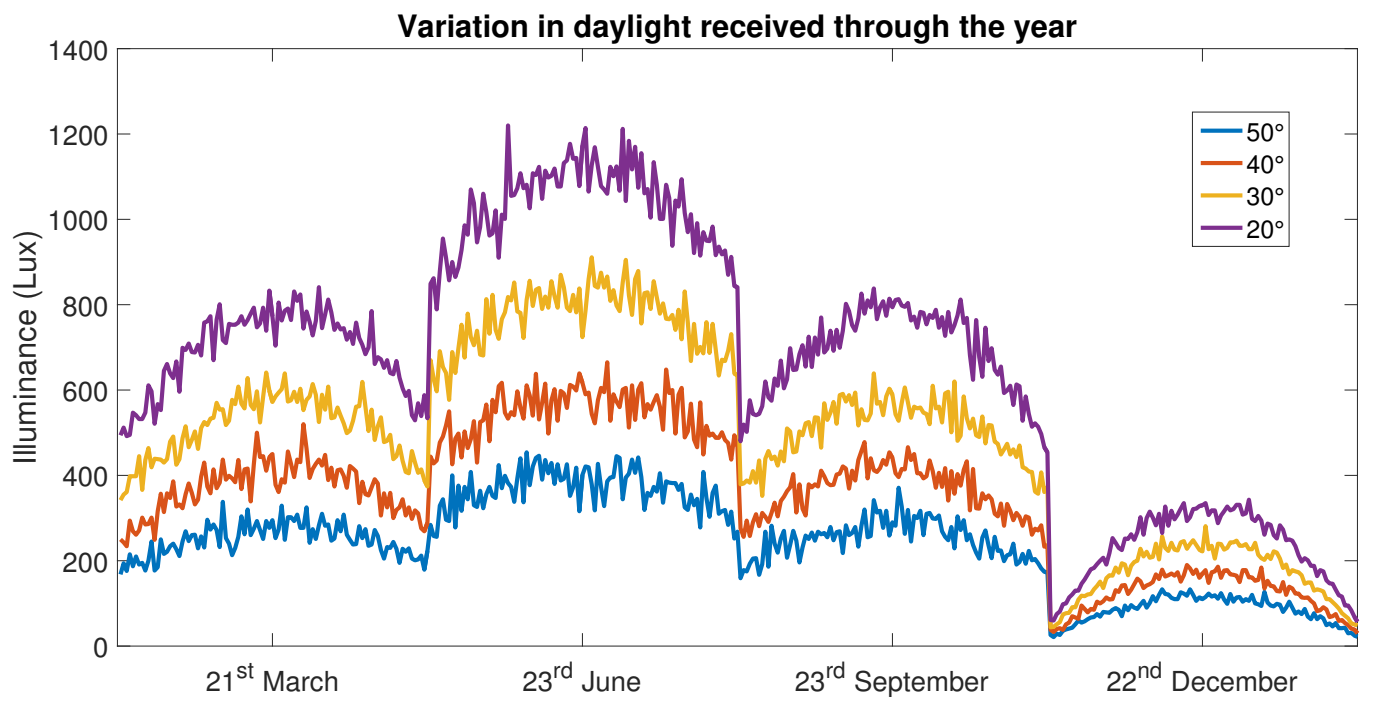

Figure 6.5: Illustration of how the received daylight varies through the year for various tilt angles.

The variation of the daylight received at a point in the center of the room, where FOS is installed, is illustrated in Figure 6.5. As previously explained in Section 4.3, this variation occurs due to the fact that the Earth is tilted on its rotational axis with respect to the orbital plane around the Sun. It also changes significantly with the latitude on the Earth's surface. These phenomena put together result in the behaviour as illustrated in the figure above. These bands of illuminance values for every season can in the future help the algorithm predict what an optimal amount of daylight that can be 
harvested. This ensures a way to pre-define the deficiency in light that has to be compensated with artificial lighting.

We use linear regression to model the variation of illuminance throughout the room, at intended locations, from sensor measurements. This enables the system to classify the type of sky condition. It was mentioned in Chapter 5 while formulating the problem that this needs to be performed in an exhaustive fashion, where the system knows the case for every combination of tilt angle and sky condition, for each day of the year.

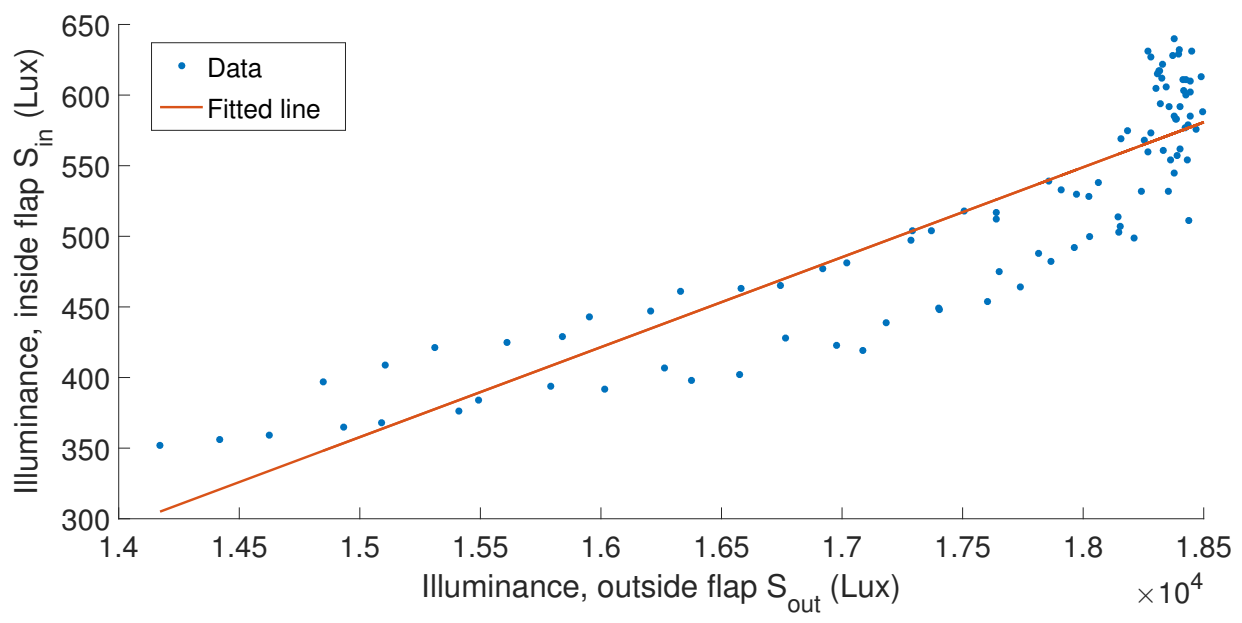

Figure 6.6: Regressional relation between $S_{\text {in }}$ and $S_{\text {out }}$ for Intermediate Sky on $23^{\text {rd }}$ June at $5^{\circ}$ tilt angle.

The plot shown in Figure 6.6 shows this relationship for a selected day with a particular sky condition and tilt angle as mentioned. $S_{\text {in }}$ is the sensor that is placed right behind the facade, inside the room and $S_{\text {out }}$ is the sensor placed on the facade. By performing exhaustive modelling, a database of these parameters can be formed, which the system can look up to ascertain the sky condition. 


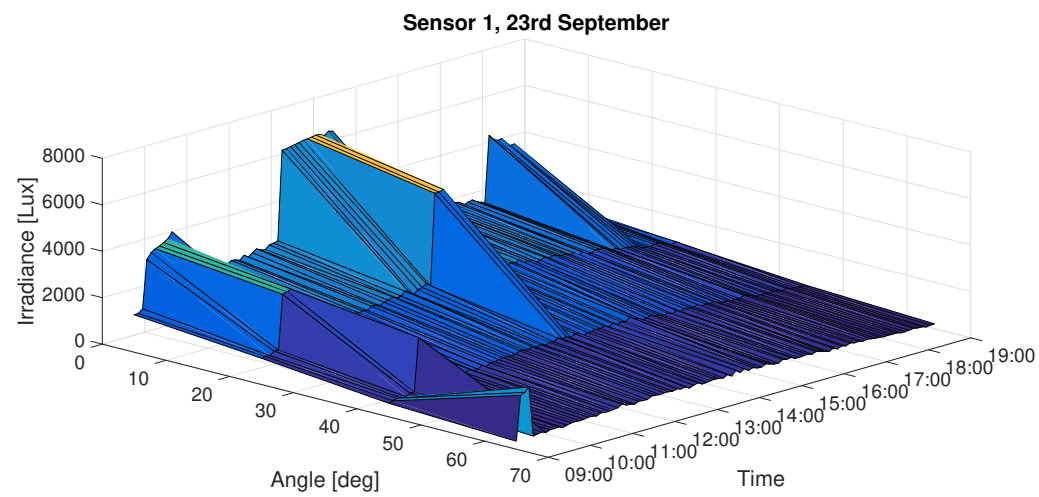

(a) Sensor 1, located at $1.475 \mathrm{~m}$ from the facade.

Sensor 2, 23rd September

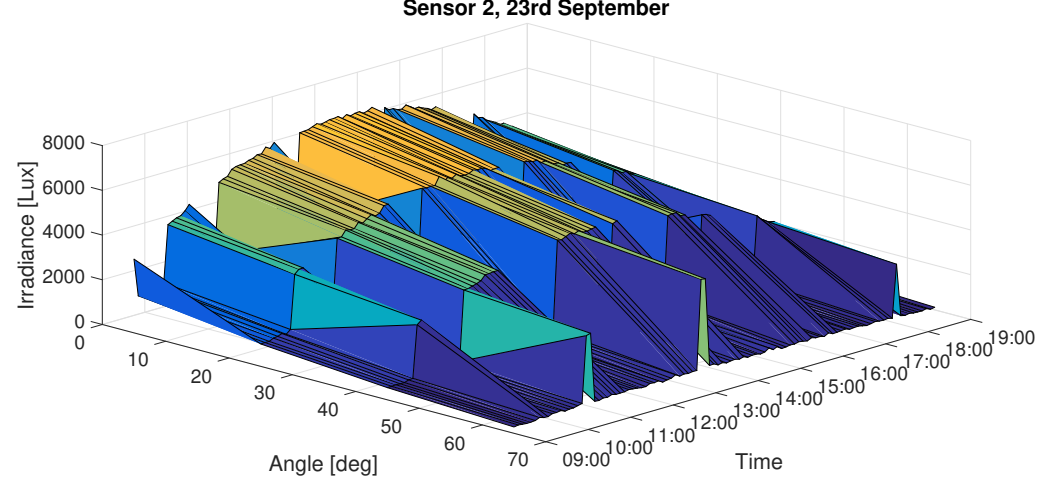

(b) Sensor 2, located at $2.95 \mathrm{~m}$ from the facade.

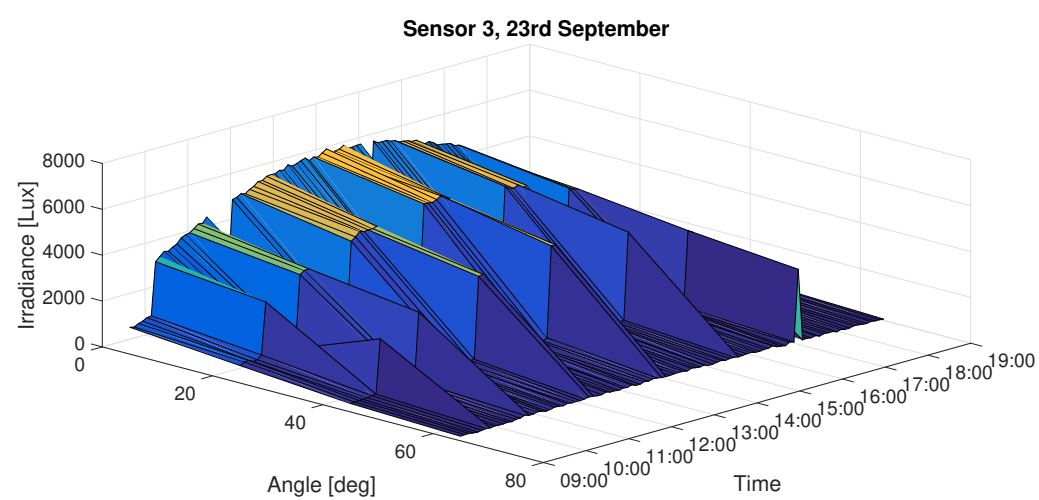

(c) Sensor 3, located at $4.425 \mathrm{~m}$ from the facade.

Figure 6.7: Comparison of illuminance data from the equi-distant sensors from the facade on a single day.

Figure 6.7 illustrates the distribution of light inside the simulated room from three different sensors on $23^{\text {rd }}$ September. The room in the simulation faces 
South, as mentioned in earlier sections, to catch the direct rays of the Sun for the entire length of the day. This, along with the fact that the sky condition chosen for this particular simulation is Clear Sky is the reason for very high values of illuminance inside for lower tilt angles. The three sensor locations inside the room are $1.475 \mathrm{~m}, 2.95 \mathrm{~m}$ and $4.425 \mathrm{~m}$ away from the facade, in the middle of the room respectively. The simulations are carried out for various tilt angles over the course of an entire day.

The three graphs help paint a picture on how light dynamics vary with different tilt angles of the facade and varying distances from the facade. The first conclusion that can be drawn from these set of graphs is that the geometric design of the facade creates pockets of high intensity within the room, otherwise called pockets of glare. This happens because the sensor is facing up, causing the direct beams of sunlight falling on it from the top, causing momentary spikes in the illuminance values. It can be seen that these spikes in iluminance values exists for certain durations of time and then drops down, with the duration decreasing with increasing tilt angle. This is caused as there is lesser gap between the flaps of the facade as the tilt angle increases, causing the moving Sun in the sky to pass over the facades. Another important conclusion that can be drawn regarding the behaviour of light under the influence of a facade indoors is that the point closest to the facade has lesser illuminance than the other two points. The point in the middle of the room has higher illuminance for a longer part of the day than the other two points. This happens as the sun is shining down at different elevations throughout the day, the direct rays that pass through the facade only reach till this point. All other points in the room are lit up because of reflected rays from the direct rays shining on the room.

A possible solution for an optimization system to avoid these spikes and direct rays of the Sun would be to mount the monitoring light sensors on the ceiling so that it always measures a combination of the direct and reflected rays in the room. This sensor value can then be mapped onto a workplane at any height from the ground using regression techniques.

Now that the light dynamics of the data from the simulations has been evaluated, we look at the energy plot for the same day. The data required is made available on special request through the PANGAEA database by KNMI [41]. The database contains the values of various radiation parameters for the location of Cabauw, $20 \mathrm{kms}$ SW of Utrecht, The Netherlands. The values used for our calculations are Direct Horizontal Irradiance and Diffuse Horizontal Irradiance over a 10-hour period. A purpose-written MATLAB code computes the energy falling on the facade over the 10-hour period using the formula explained in section 2 . 


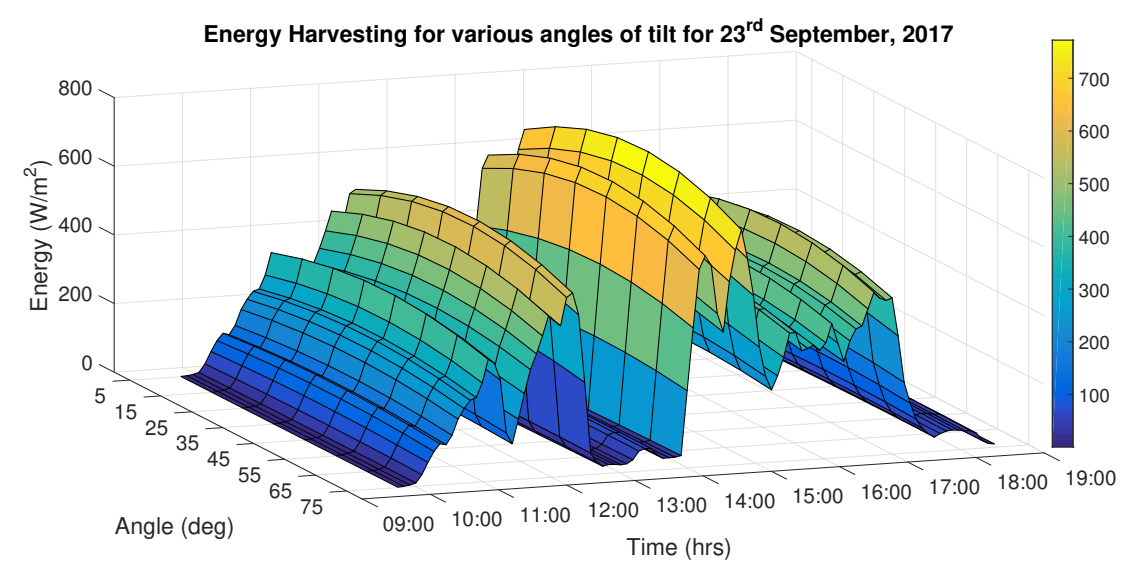

Figure 6.8: Available energy for harvesting at various angles of tilt.

The plot, illustrated in Figure 6.8, shows the radiation available to the solar cells on the facade through the course of the day for various values of tilt angle. From the plot, it can be inferred that the amount of energy available on the facade increased steadily till about $12 P M$. It then drops suddenly to a very low value, suggesting that it was cloudy, for about the next two hours. The plot then shows that it was sunny for the next one hour, followed by a small turbulence and gradually drops as the sun begins to set in the evening, showcasing a perfect example of Dutch weather. At every measured instant, the amount of energy available also varies with the tilt angle in an arc-like fashion, suggesting that certain angles have the potential to harvest more energy than others.

In order to correlate the illuminance data from the simulations and energy data from the KNMI database, a MATLAB code is necessary as the frequency at which data was logged was different for both the datasets. Now that we have evaluated the data from the simulations as well as the energy available with the KNMI datasets, we look at the proposed algorithm iFOS and some use cases. 


\subsection{Proposed Algorithm: iFOS}

This section aims to evaluate the proposed algorithm iFOS through multiple use cases. However, before iFOS is evaluated, we want to create two corner cases, which would as benchmarks to compare iFOS against. The comfort of the user and the amount of energy harvested are the parameters the system optimizes. Hence, we evaluate two benchmarks, one where the system works to maximize comfort at all times and another where the system works to maximize energy harvested at all times. This is equivalent to adding a full weight to each of the parameters to be optimized. We then study how the system works with the proposed iFOS algorithm and compare its performance with the two benchmarks. We also evaluate the variation in performance of the iFOS algorithm at various levels of constraints. The day chosen for these evaluations is March $21^{\text {st }}$ with Intermediate Sky condition. The energy data for the same day is downloaded from the KNMI database. Both the datasets are also adjusted and correlated in time as they have different frequencies. All the evaluations are performed for the comfort set at medium level, as explained in the previous chapter. This means that the illuminance is maintained in the range of $[400,600]$ lux. Comparisons for the performance of the algorithm at the other comfort levels are provided in the appendix.

\subsubsection{Benchmark 1: Maximum Comfort}

One of the main functions of FOS is to ensure high levels of comfort for the inhabitants of the building. As seen in the formulation in Section 5, the comfort is defined by a metric "c", called the Comfort Number, defined by:

$$
c=\frac{l_{o p t}}{\left|l_{o p t}-l_{\text {in }}\right|}
$$

where, $l_{\text {opt }}$ is set to 500 , as research suggested.

We set $l_{\text {in }}$ to be in between $[400,600]$. This means that in the equation, $c$ takes a minimum value of 5 . Hence,

$$
c \geq 5
$$

At every instant, the algorithm simply picks the tilt angle that gives the maximum comfort within the defined range. The energy harvested is what is available at that tilt angle ensuring maximum comfort, i.e. we maximize comfort number. There may arise a case that the $l_{\text {in }}$ is 500 , where the comfort becomes $\infty$. Here, we simply assume the comfort number to take 500 as its value. 


\section{Results}

Figure 6.9 illustrates the behaviour of the system in Maximum Comfort mode. As the graph suggests, the comfort is always maintained at the maximum possible level, with the second graph showing the corresponding energy available to be harvested. The third plot shows the number of times the facade has to change the current tilt angle in order to maintain maximum comfort at all times. This can be thought of as context switching, which consumes energy as the motors controlling the facade need to be run.
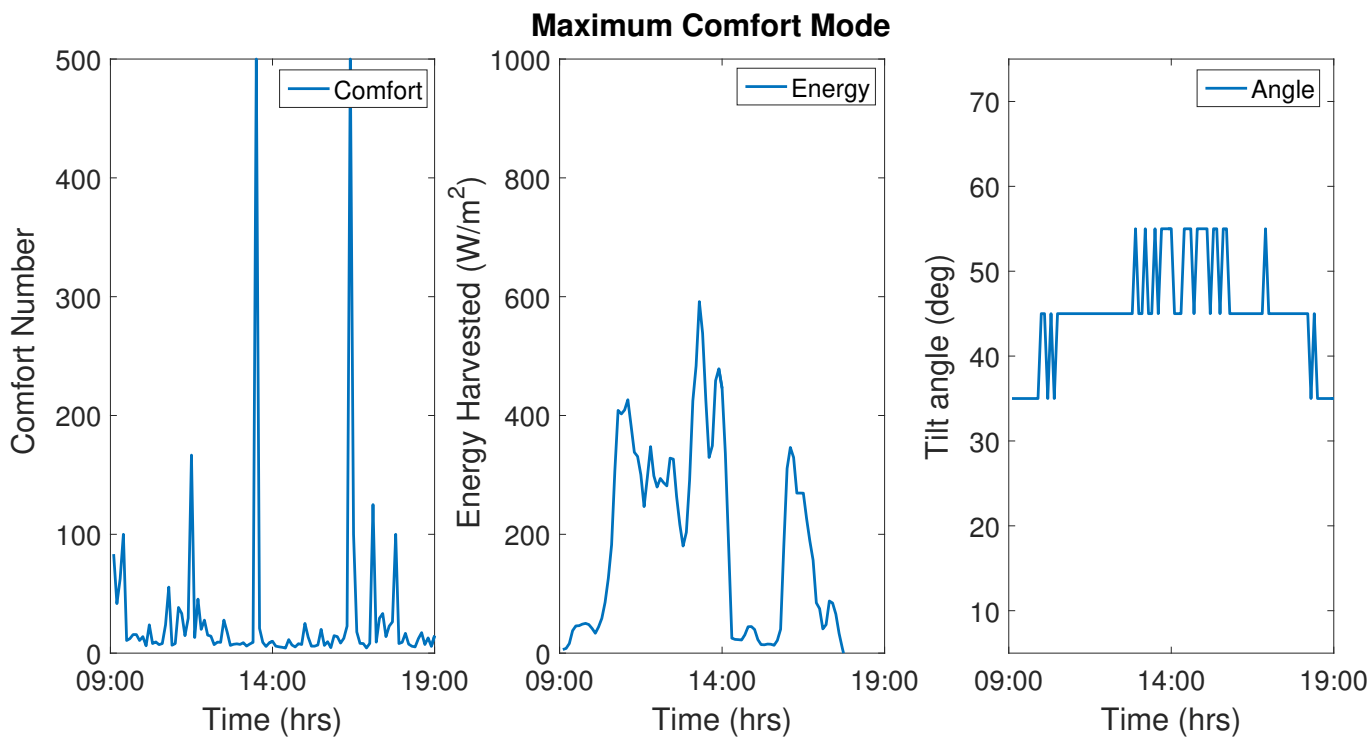

Figure 6.9: Plot of Comfort Number, Energy Harvested and Tilt angle over the entire day in Maximum Comfort mode. 


\subsubsection{Benchmark 2: Maximum Energy Harvested}

The second benchmark is the case when the system works to maximize the energy harvested by the solar cells on the facade. In optimization terms, the energy takes one full weight in this setting. Here, the algorithm computes the energy harvesting potential for each tilt angle of the facade. Then, the tilt angle that has the potential to harvest maximum energy is chosen and the user only receives the corresponding comfort level.

\section{Results}

The behaviour of the system when set to Maximum Energy mode is illustrated in Figure 6.10. The Comfort Number is almost always at the lowest possible value, around 5 . The energy plot has the same pattern as the previous case but the magnitude at every instant is higher, suggesting that more energy is harvested through the day. The tilt angle plot shows fewer variations, suggesting that to maintain the system in Maximum Energy mode, lesser number of changes are required to be made through the course of the day.
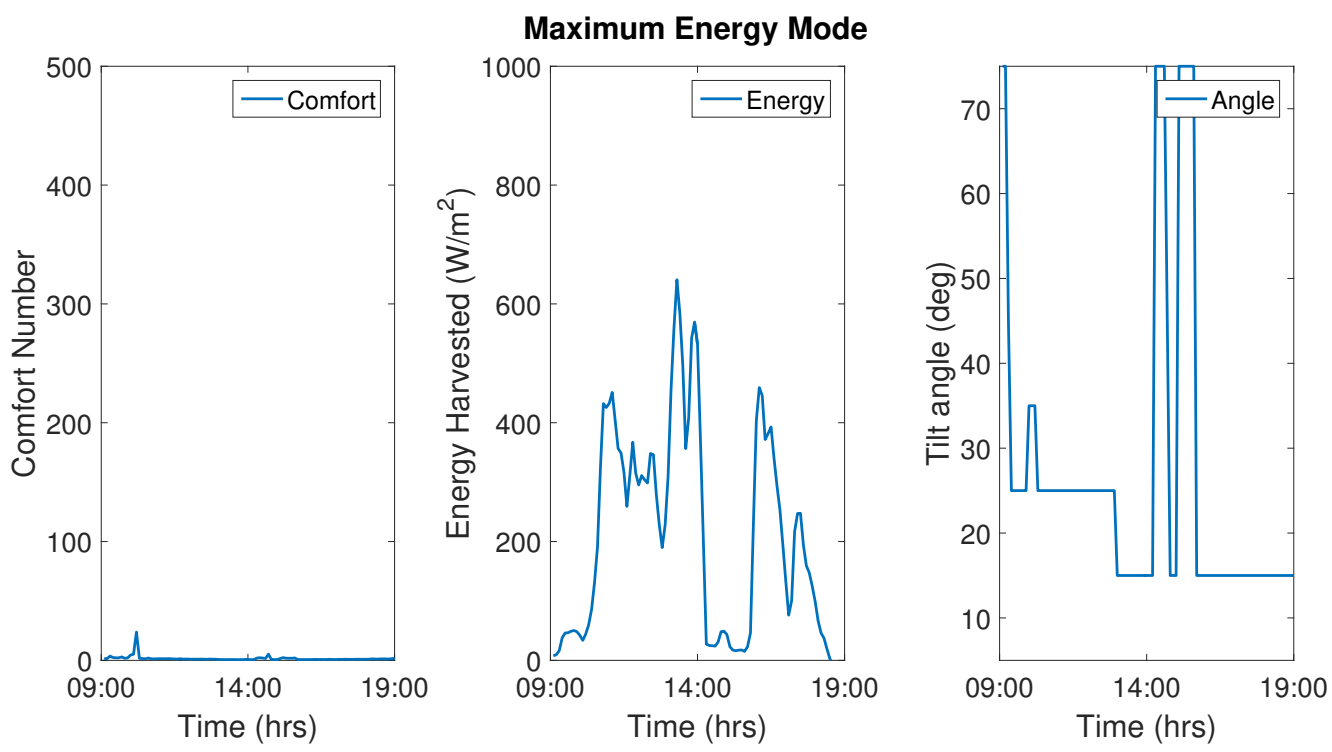

Figure 6.10: Plot of Comfort Number, Energy Harvested and Tilt angle over the entire day in Maximum Energy mode. 


\subsection{3 iFOS Optimized}

The extent to which the proposed algorithm optimizes the comfort and energy harvesting of the facade depends on the range in which the indoor illuminance $l_{\text {in }}$ is to be maintained. Since both the previous benchmarks are set for $l_{i n} \in[400,600]$, we will evaluate the algorithm also for the same requirements indoors. iFOS optimizes the problem by first taking the Comfort Number above the set threshold and then maximizing the energy harvesting potential from the set of available Comfort Numbers.

\section{Results}

The behaviour of the system in iFOS Optimized mode is illustrated in Figure 6.11. The Comfort Number plot shows a considerable amount of comfort through the day while the differences in energy harvested are better than the ones in Maximum Comfort at some points through the day, varying between $4 \%$ to $66 \%$ instantaneously. The tilt angle, changing 21 times during the course of the day, has lesser variation than Maximum Comfort, which varies 26 times but more than Maximum Energy, which varies just 8 times.
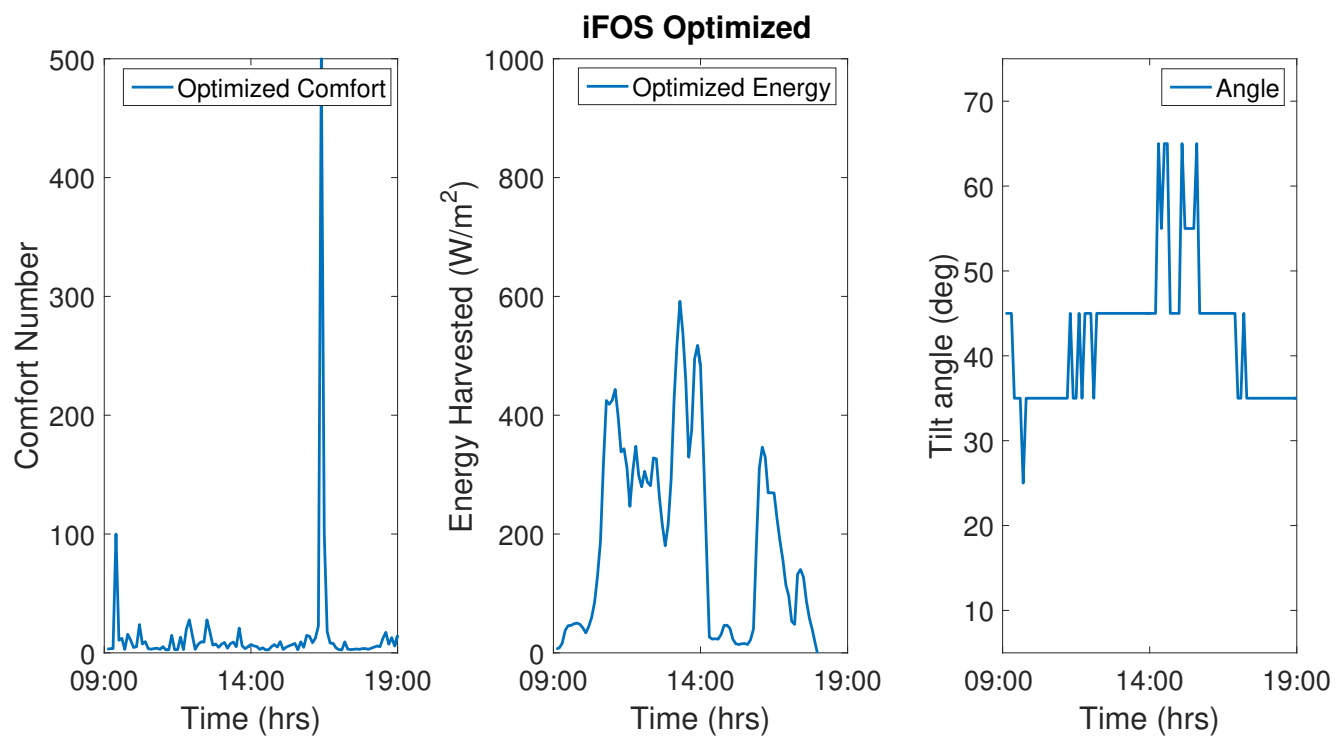

Figure 6.11: Plot of Comfort Number, Energy Harvested and Tilt angle over the entire day in iFOS Optimized mode. 


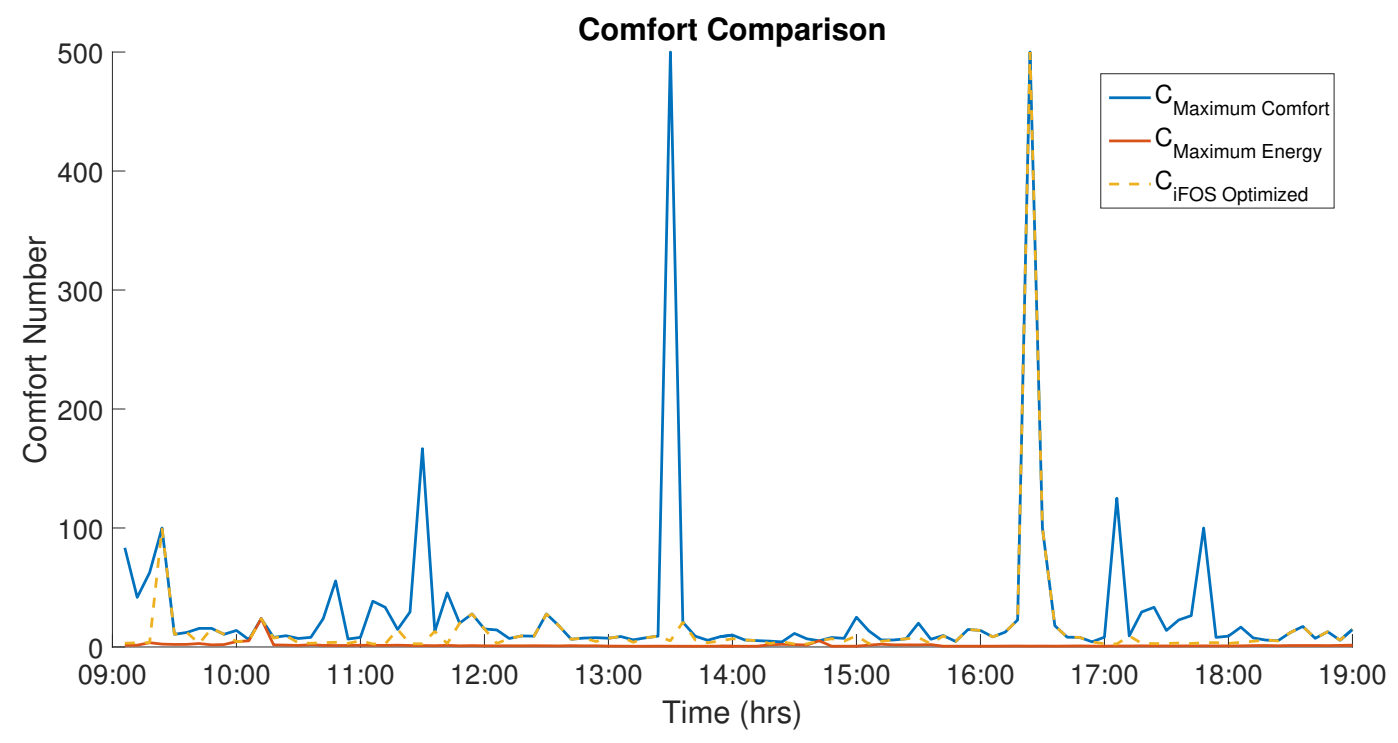

Figure 6.12: Comparing the Comfort Number of the two benchmarks with iFOS.

To get a better view of the iFOS Optimized improvements, we plotted them together. Figure 6.12 shows the comparison of the Comfort Number of both the benchmarks and the iFOS optimized system. For most parts of the day, the iFOS optimized system maintains as much comfort as the Maximum Comfort system. At all times, it maintains higher comfort than the Maximum Energy system.

Figure 6.13 compares the potential energy that can be harvested for the two benchmarks and the iFOS system. For most parts of the day, the iFOS system has a superior energy harvesting potential that the Maximum Comfort system. Two instants in particular are marked, Instant ${ }_{1}$ and Instant ${ }_{2}$, at different times of the day. Instant $t_{1}$ has a $4 \%$ increase in energy harvesting potential over the Maximum Comfort system with a 34 lux drop in the illuminance indoor. Instant 2 offers an $8 \%$ increase in the energy harvesting potential over the Maximum Comfort with a 41 lux drop in the illuminance indoor. The actual impact of this increase in the potential to harvest energy is discussed in the following subsection. By taking the area under the curves, for an entire day, the iFOS optimized algorithm provides $3.2 \%$ increase in the energy harvested over the Maximum Comfort benchmark, while ensuring similar comfort $60 \%$ of the time. The comparisons for other values of "c" are provided in the Appendix. 


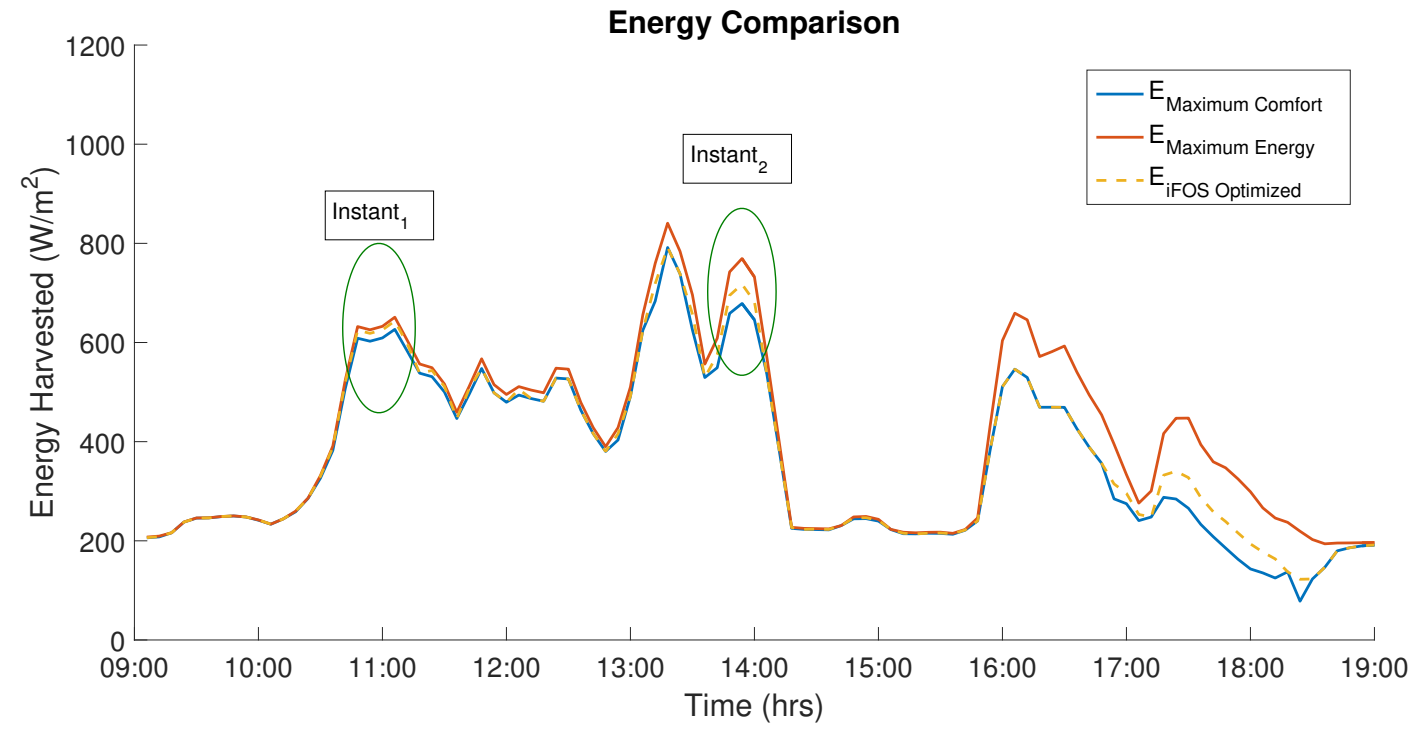

Figure 6.13: Comparing the energy harvesting potential of the two benchmarks with iFOS.

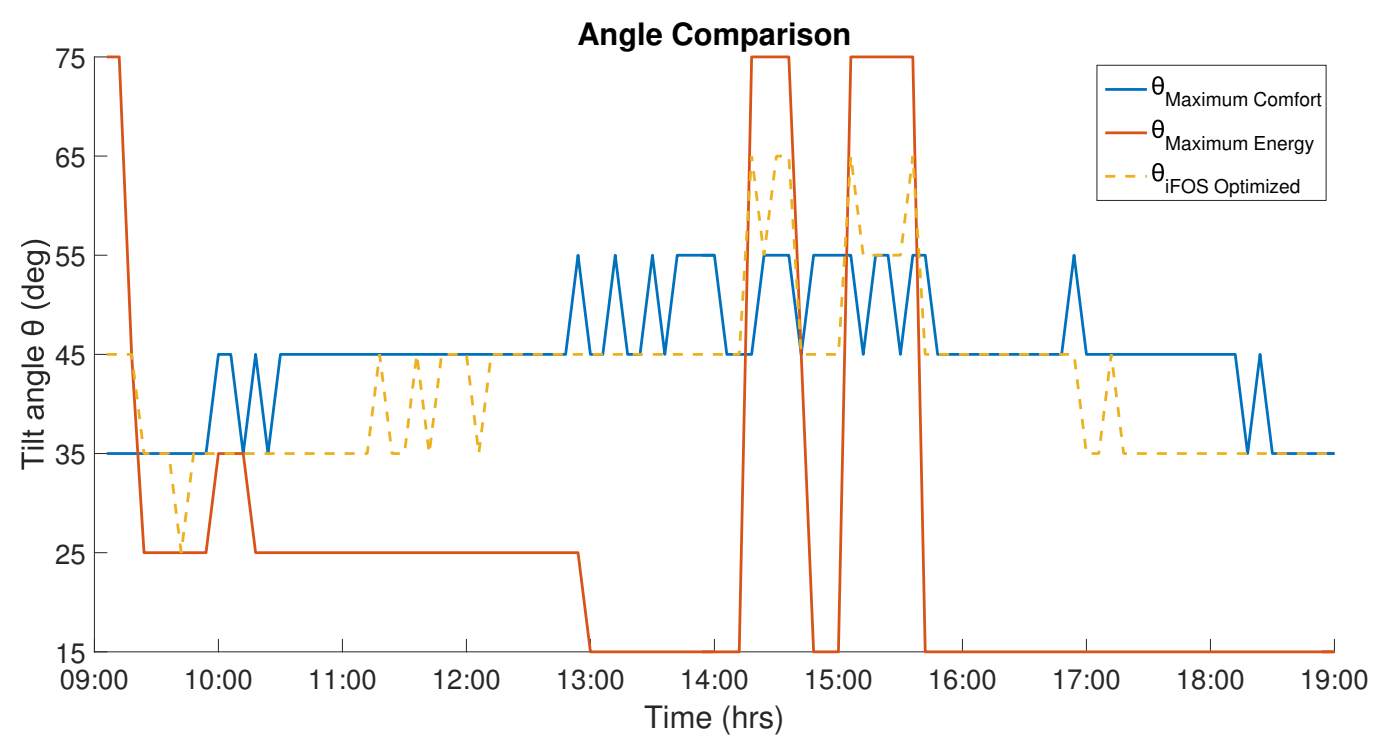

Figure 6.14: Comparing the switching of tilt angles of the two benchmarks with iFOS.

It is discussed previously that the number of times the system changes the tilt angle in a day can have an impact on its energy signature as energy is 
spent in running the motors to make the adjustments, also causing inconveniences to the user. In this direction, it is critical to analyze the number of times each system makes a switch. Figure 6.14 shows the extent of switching of the tilt angle for both the benchmarks and iFOS. From the plot, it can be concluded that the highest energy expenditure occurs for the Maximum Comfort mode, as it switches the tilt angle more frequently in comparison to the other two. The Maximum Energy system switches the least and iFOS switches the tilt angle more frequently that Maximum Energy but less frequently than Maximum Comfort. Further analysis beyond the scope of this research work needs to be done to ascertain the exact amount of energy expenditure in these scenarios as the switching occurs from various angles. In some cases, the switching that needs to occur is about $50^{\circ}$, which may consume more power than a switch of $10^{\circ}$.

Now that we have evaluated the behaviour of these systems, the next subsection aims to put things into perspective with numbers and how much of an impact iFOS actually makes in comparison.

\subsubsection{Impact of iFOS}

To fully comprehend the effects of the increase in energy harvesting with iFOS, the concept needs to be looked at as a scaled-up system. For this, first we convert the $W / \mathrm{m}^{2}$ values into the more standard $\mathrm{kWh} / \mathrm{m}^{2} .1 \mathrm{kWh} / \mathrm{m}^{2}$ is the amount of energy harvested in 1 hour from a solar cell of area $1 \mathrm{~m}^{2}$. As shown in Section 3.1, the total area covered by solar cells on a facade outside a single window is $1.6 \mathrm{~m}^{2}$ and one whole side of the building is 750 $m^{2}$.

The total amount of energy harvested for the evaluated day is calculated by taking the area under the energy curve. For iFOS optimized algorithm, the figure was $75.0017 \mathrm{~W} / \mathrm{m}^{2}$, which is about $1.8 \mathrm{kWh} / \mathrm{m}^{2} /$ day. With a $20 \%$ efficiency in conversion, the whole one side of the building captures about $270 \mathrm{kWh}$ of solar radiation on that day. This is enough energy to run about 2700 LEDs rated at $10 \mathrm{~W}$ for 10 hours or fully charge 5 electric cars, each with a $50 \mathrm{kWh}$ battery, at the end of the day.

The average energy figure for The Netherlands in the summer months is about $5 \mathrm{kWh} / \mathrm{m}^{2} /$ day, which makes the total energy that can be captured at $20 \%$ efficiency to be about $750 \mathrm{kWh}$ per day. 
The iFOS optimized algorithm has the potential to harvest about $5 \mathrm{~W} / \mathrm{m}^{2}$ more energy that the Maximum Comfort algorithm on the measured day. This translates to nearly $18 k W h$ more energy at the end of the day than the Maximum Comfort algorithm. When there are no occupants detected in the room, the system switches itself to the Maximum Energy mode, during which, it gives one full weight to energy harvesting in the optimization problem. 


\section{Chapter 7}

\section{Conclusion and Future Work}

A considerable amount of energy can be harvested from the sides of high-rise buildings while ensuring sufficient comfort levels for its inhabitants. This thesis presents a concept called "FOS", that optimizes the user comfort while maximizing energy harvested from the sides of high-rise buildings. The system is conceptualized and designed using a software called Rhinoceros, with other plug-ins.

A simulated test bed is used to gather data for various days across the year and under varying sky conditions. A small-scale test bed was built from scratch in an identical room to gather real-world data and compare it to the simulated data. An average R-squared value of 0.802 was achieved between real and simulated data, proving the validity of the simulations. Data is logged to understand the dynamics of light indoors, at different tilt angles of the facade. A purpose-written MATLAB code computes the potential energy that could be harvested through the day at different tilt angles. An optimization problem is formulated for simultaneously optimizing the user comfort and maximizing energy harvested from the facade. A metric called "Comfort Number" is defined as a measure of how close the indoor illuminance level is to the optimal illuminance level. An optimization algorithm called "iFOS" is proposed, that controls the movement of the facade, optimizing user comfort and maximizing energy harvested. It was evaluated against two benchmarks: one optimizing for Maximum Comfort and the other for Maximum Energy. The algorithm shows instantaneous improvements in the energy harvested, varying from $4 \%$ to $66 \%$ while the providing lesser comfort. Evaluations also suggest that the algorithm performs the best when working within the range of $[400,600]$ lux. 
Figures suggest that for The Netherlands, such a conceptual system, with about $20 \%$ conversion efficiency of the solar cells, can harvest about $750 \mathrm{kWh}$ from a $750 \mathrm{~m}^{2}$ area of solar cells. Although the figures of energy suggest significant figures, an implementation of this system to scale would cost a substantial amount of investment as solar cells are still on the expensive side. Such a system would then transfer the energy harvested back into the grid, and this requires power analysis and other computations, taking into account conversion efficiency of these processes. The authors are quite convinced that energy-harvesting skins for buildings have a great potential in building self-sustaining urban centers of the future. That said, it is along way ahead before these concepts become a reality.

As mentioned earlier, this work is a preliminary exploration into the concept and there is still a lot that is unknown, a lot of assumptions that were considered and a few parameters that were not taken into account. These could possibly be starting points for anyone looking to further explore this concept. Prediction of cloud movements can help the system optimize its movements, saving energy in the process. The facades were placed facing South for the entire duration of the simulation. Other orientations could be tested to give an overview of which orientations are feasible for the system. The effect of shading can be considerable, when a layer of the facade casts a shadow on the lower layers. This possibility was not taken into account in the computations and can give a more accurate insight into the figures for energy.

The ideal test bed to learn about the system would be a full-scale room with a facade fixed outside the window. This enables a dataset that gives illuminance values and their corresponding solar radiation components, which can provide a better understanding on the intricate relationship between light and energy. This was not possible through simulated data. Concluding, this concept proves to have immense potential in unlocking the power of the Sun and can boost the possibilities of off-grid energy solutions in the ever-growing urban centers of the world. 


\section{Appendix A}

\section{Comparison plots for $\mathrm{c}=10$}

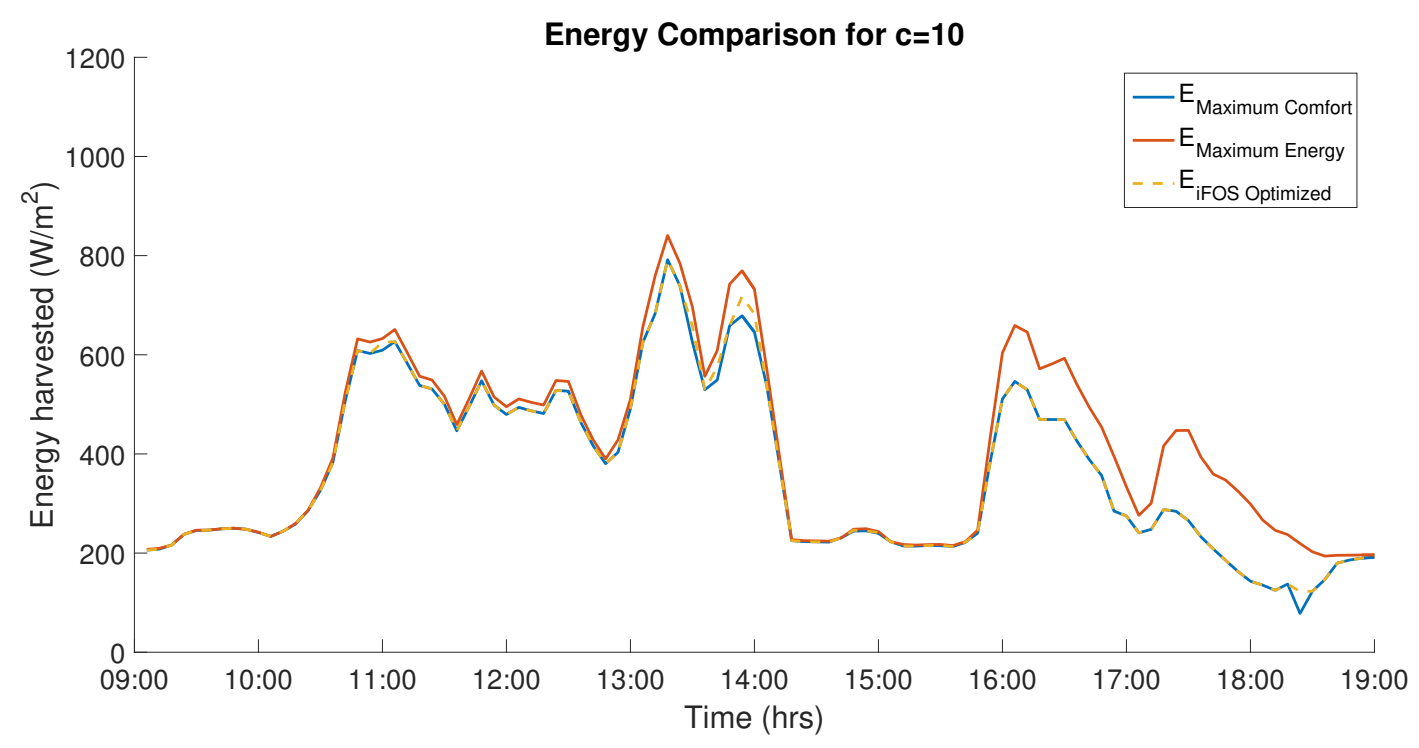

Figure A.1: Comparing the energy harvesting potential of the two benchmarks with iFOS.

Over the course of the day, the iFOS optimized algorithm harvest $0.63 \%$ more energy than the maximum comfort algorithm while ensuring similar comfort $90 \%$ of the time. 


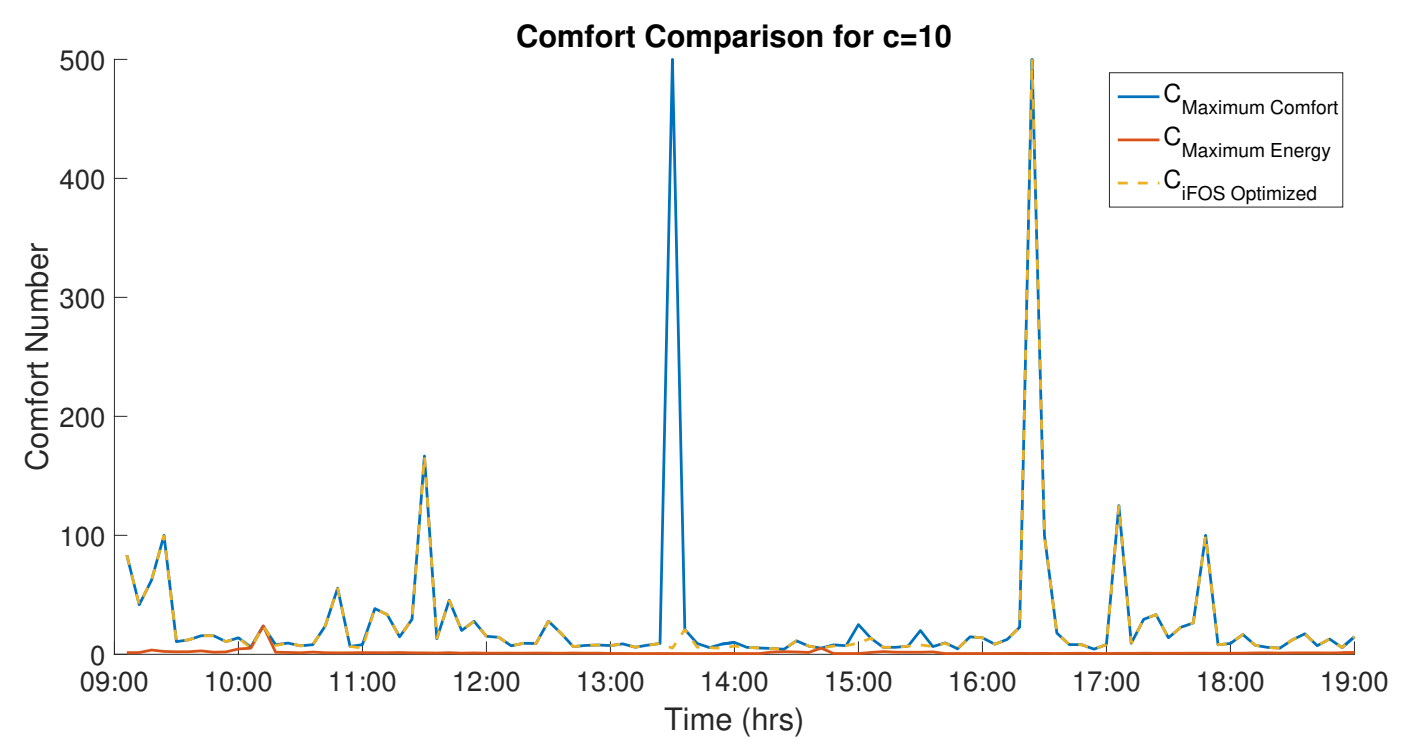

Figure A.2: Comparing the Comfort Number of the two benchmarks with iFOS.

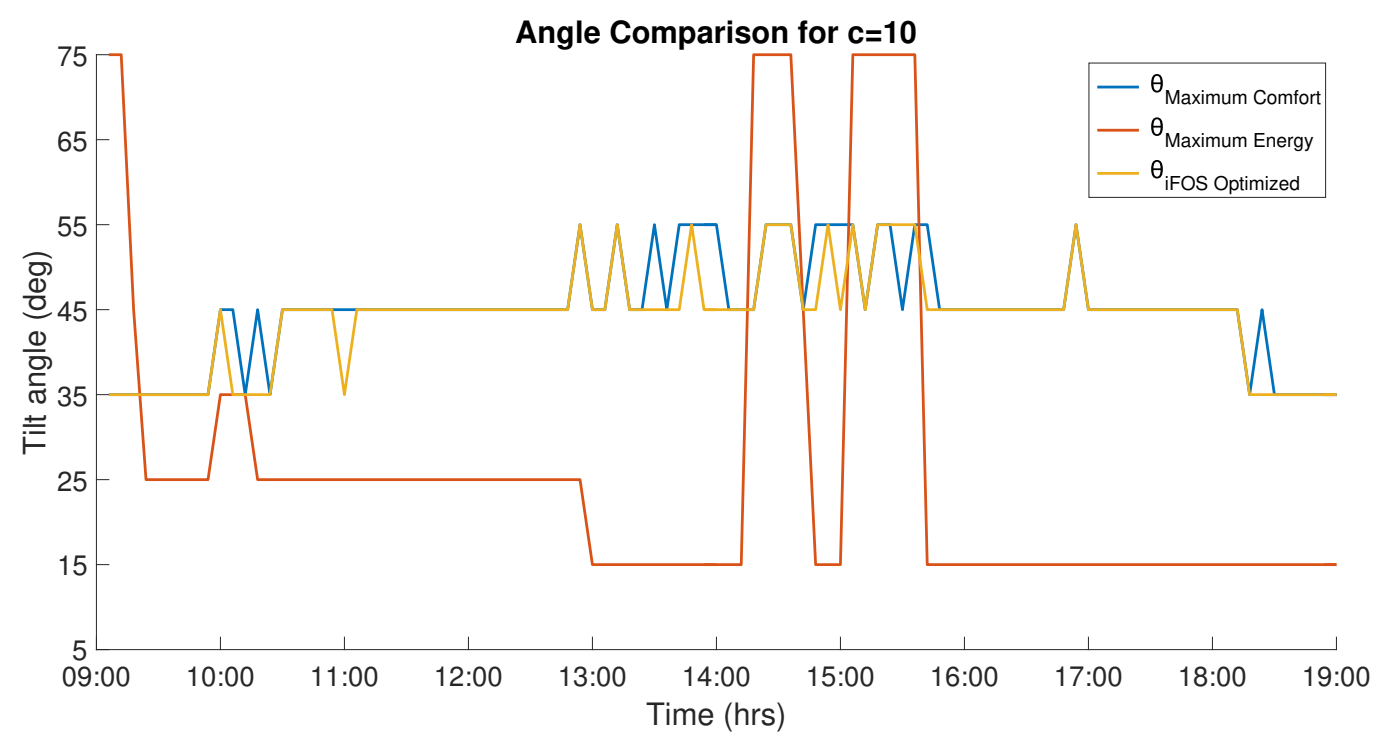

Figure A.3: Comparing the switching of the tilt angle of the two benchmarks with iFOS. 


\section{Appendix B}

\section{Comparison plots for $\mathrm{c}=2.5$}

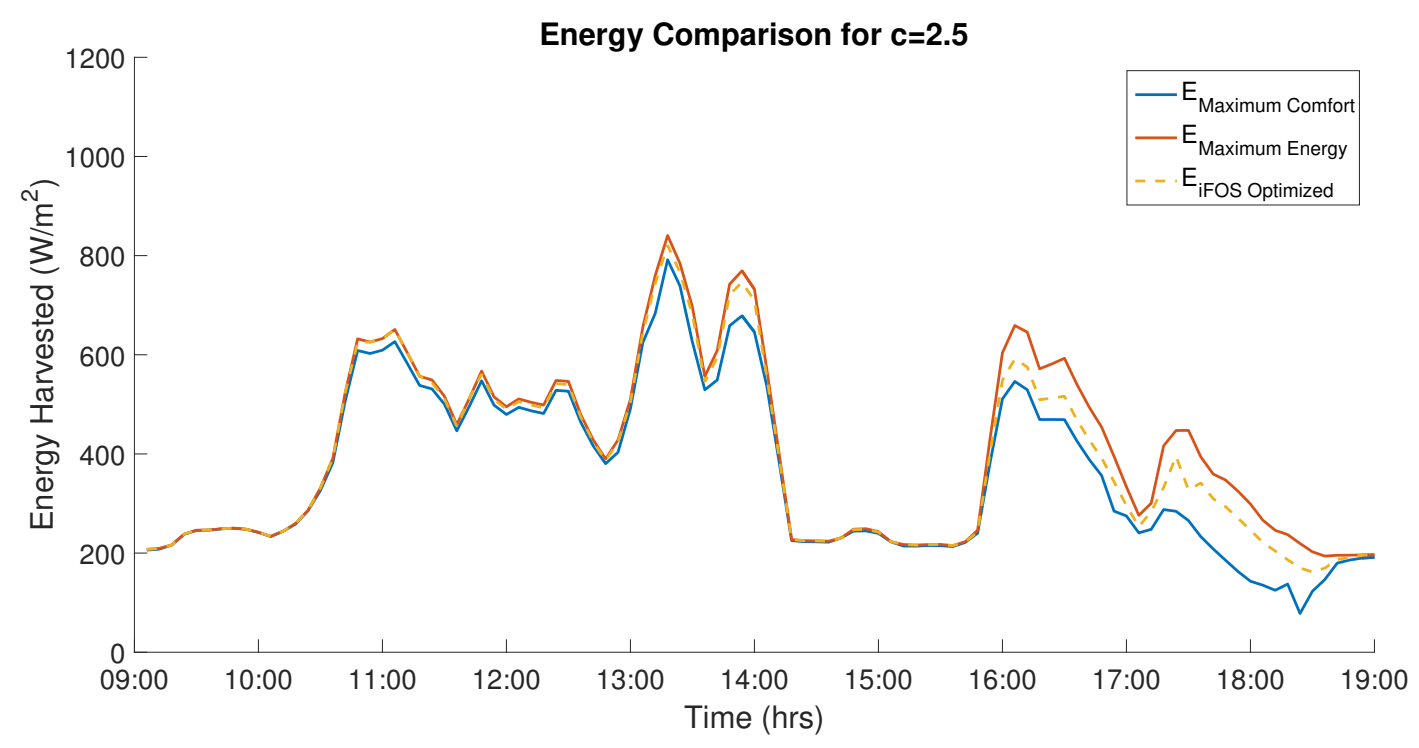

Figure B.1: Comparing the energy harvesting potential of the two benchmarks with iFOS.

Over the course of the day, the iFOS optimized algorithm harvest $9 \%$ more energy than the maximum comfort algorithm while ensuring similar comfort $10 \%$ of the time. 


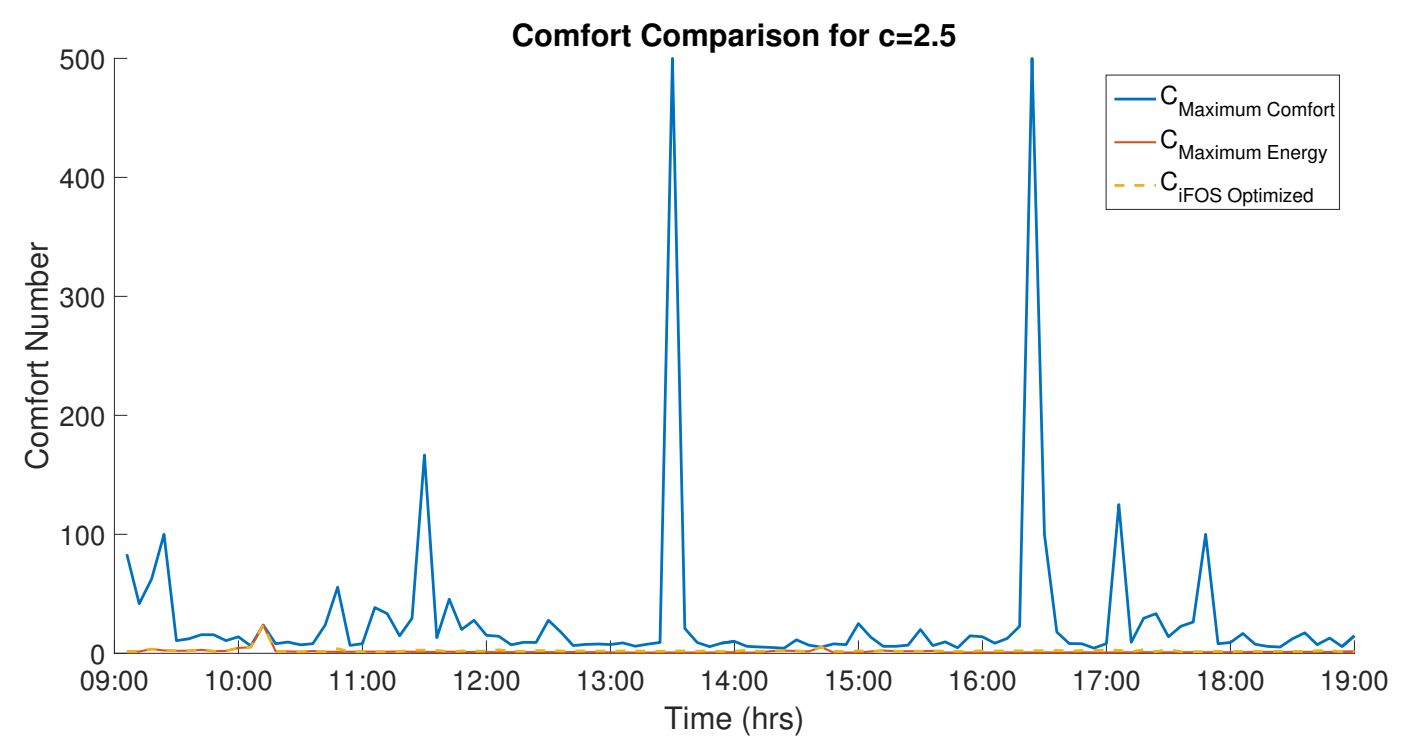

Figure B.2: Comparing the Comfort Number of the two benchmarks with iFOS.

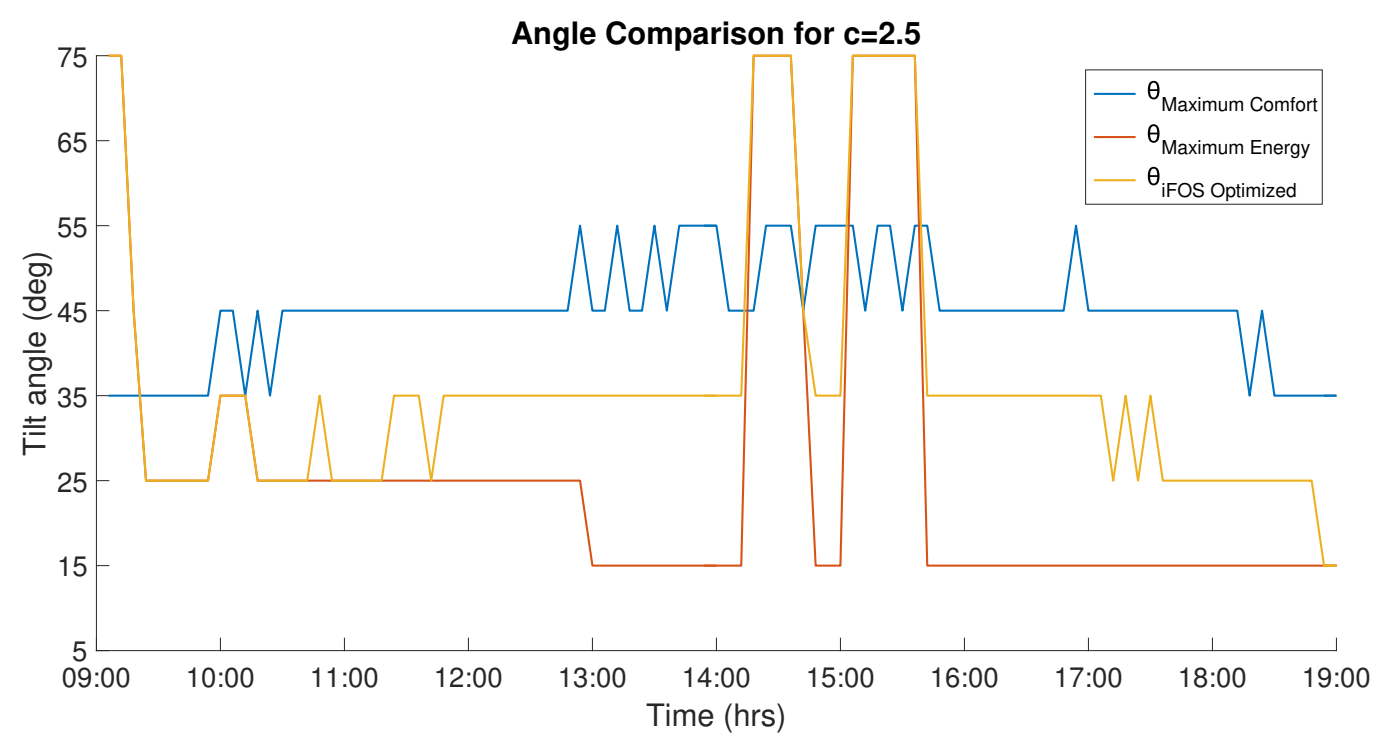

Figure B.3: Comparing the switching of the tilt angle of the two benchmarks with iFOS. 


\section{Bibliography}

[1] Mir M Ali and Kheir Al-Kodmany. Tall buildings and urban habitat of the 21st century: a global perspective. Buildings, 2(4):384-423, 2012.

[2] Karin Fridell Anter. DAYLIGHT: Visual Comfort and Quality of Light. SYN-TES Report, 2013.

[3] Christoph F Reinhart and Oliver Walkenhorst. Validation of dynamic radiance-based daylight simulations for a test office with external blinds. Energy and buildings, 33(7):683-697, 2001.

[4] National renewable energy laboratory. https://www.nrel.gov, 2016.

[5] Trends in Global CO2 Emissions, 2016 Report. PBL Netherlands Environmental Assessment Agency, Joint Research Center: European Commission, 2017.

[6] Solar is the fastest-growing energy. https://www.bloomberg.com, 2016.

[7] Changhai Peng, Ying Huang, and Zhishen Wu. Building-integrated photovoltaics (bipv) in architectural design in china. Energy and Buildings, 43(12):3592-3598, 2011.

[8] Luis Pérez-Lombard, José Ortiz, and Christine Pout. A review on buildings energy consumption information. Energy and buildings, 40(3):394398, 2008.

[9] Sarah Stevens. Intelligent façades: Occupant control and satisfaction. International journal of solar energy, 21(2-3):147-160, 2001.

[10] Geun Young Yun, Mike McEvoy, and Koen Steemers. Design and overall energy performance of a ventilated photovoltaic façade. Solar Energy, 81(3):383-394, 2007.

[11] Brandon C Andow, Bess Krietemeyer, Peter RH Stark, and Anna H Dyson. Performance criteria for dynamic window systems using nanostructured behaviors for energy harvesting and environmental comfort. In Proc. of SPIE Vol, volume 8692, pages 86923V-1, 2013. 
[12] Alessia Giovanardi. Integrated solar thermal facade component for building energy retrofit. PhD thesis, University of Trento, 2012.

[13] Jenny Lovel. Building envelopes: an integrated approach. Princeton Architectural Press, 2013.

[14] Dudley McFarquhar. The Role of the Building Facade: Curtain Walls. Building Enclosure Science and Technology Conference, McFarquhaer Group Inc., Building Enclosure Technology and Environment Council, 2012.

[15] RCGM Loonen, M Trcka, and J Hensen. Exploring the potential of climate adaptive building shells. In Building Simulation 2011: 12th Conference of International Building Performance Simulation Association,(14-16 November), pages 2148-2155, 2011.

[16] Raymond J Cole, Audrey Bild, and Esteban Matheus. Automated and human intelligence: direct and indirect consequences. Intelligent Buildings International, 4(1):4-14, 2012.

[17] Seokyoun Kang, Taeyon Hwang, and Jeong Tai Kim. Theoretical analysis of the blinds integrated photovoltaic modules. Energy and Buildings, 46:86-91, 2012.

[18] Jiakang Lu, Dagnachew Birru, and Kamin Whitehouse. Using simple light sensors to achieve smart daylight harvesting. In Proceedings of the 2nd ACM Workshop on Embedded Sensing Systems for EnergyEfficiency in Building, pages 73-78. ACM, 2010.

[19] Rohit Kumar. New algorithms for daylight harvesting in a private office. In Information Fusion (Fusion), 2015 18th International Conference on, pages 383-392. IEEE, 2015.

[20] Yuan Wang and Partha Dasgupta. Designing an adaptive lighting control system for smart buildings and homes. In Networking, Sensing and Control (ICNSC), 2015 IEEE 12th International Conference on, pages 450-455. IEEE, 2015.

[21] Younju Yoon, Jin Woo Moon, and Sooyoung Kim. Development of annual daylight simulation algorithms for prediction of indoor daylight illuminance. Energy and Buildings, 118:1-17, 2016.

[22] Sandia National Laboratories: National Technology and Engineering Solutions of Sandia LLC 2014. Pv performance modelling collaborative.

[23] Ibrahim Reda and Afshin Andreas. Solar position algorithm for solar radiation applications. Solar energy, 76(5):577-589, 2004. 
[24] Kalyanmoy Deb. Multi-objective optimization using evolutionary algorithms, volume 16. John Wiley \& Sons, 2001.

[25] Robert McNeel et al. Rhinoceros. NURBS modelling for Windows: http://www. rhino3d. com/jewelry. htm, 2015.

[26] Robert McNeel et al. Grasshopper generative modeling for rhino. Computer software (2011b), http://www. grasshopper3d. com, 2010.

[27] LLC Solemma. Diva for rhino. Available::[accessed 27.06. 15], 2014.

[28] Dr R Compagnon. Radiance: a simulation tool for daylighting systems. The Martin Centre for Architectural and Urban Studies University of Cambridge Department of Architecture, 1997.

[29] CF Reinhart. Daysim. Accessed June, 12, 2011.

[30] Time and Date AS. https://www.timeanddate.com, 2015.

[31] Koninklijk nederlands meteorologisch instituut. https://www.knmi.nl, 2015.

[32] Wouter Knap. Basic and other measurements of radiation at station Cabauw (2017-03), 2017.

[33] Poul O Fanger et al. Thermal comfort. analysis and applications in environmental engineering. Thermal comfort. Analysis and applications in environmental engineering., 1970.

[34] J Fergus Nicol and Michael A Humphreys. Adaptive thermal comfort and sustainable thermal standards for buildings. Energy and buildings, 34(6):563-572, 2002.

[35] Richard J De Dear and Gail S Brager. Thermal comfort in naturally ventilated buildings: revisions to ashrae standard 55. Energy and buildings, 34(6):549-561, 2002.

[36] Joan M Daisey, William J Angell, and Michael G Apte. Indoor air quality, ventilation and health symptoms in schools: an analysis of existing information. Indoor air, 13(1):53-64, 2003.

[37] Francesco Martellotta, Alessandro Cannavale, Michele DAlba, Sabrina Della Crociata, and Antonio Simone. Optimization of indoor environment quality for hypermarket workers: from subjective response to objective design criteria. Energy Procedia, 101:272-279, 2016.

[38] Fabio Sicurella-Planair SA-fabio. Passive solutions for the optimization of the indoor environmental quality: a case study. 
[39] Jun Mei, Bing Zhu, and Xiaohua Xia. Model predictive control for optimizing indoor air temperature and humidity in a direct expansion air conditioning system. In Control and Decision Conference $(C C D C)$, 2015 27th Chinese, pages 2491-2496. IEEE, 2015.

[40] Chayan Sarkar, SN Akshay Uttama Nambi, and R Venkatesha Prasad. iltc: Achieving individual comfort in shared spaces. In EWSN, pages 65-76, 2016.

[41] Wouter Knap. Basic and other measurements of radiation at station Cabauw (2017-09), 2017. 